\title{
Passive Detection of Moving Aerial Target Based on Multiple Collaborative GPS Satellites
}

\author{
Mingqian Liu ${ }^{1}$, Zhiyang Gao ${ }^{1, *}$, Yunfei Chen ${ }^{2}$, Hao Song ${ }^{3} \odot$ and Yuting Li $^{1}$ \\ and Fengkui Gong ${ }^{1}$ \\ 1 State Key Laboratory of Integrated Service Networks, Xidian University, Xi'an 710071, China; \\ mqliu@mail.xidian.edu.cn (M.L.); mqliu@xidian.edu.cn (Y.L.); fkgong@xidian.edu.cn (F.G.) \\ 2 School of Engineering, University of Warwick, Coventry CV4 7AL, UK; yunfei.chen@warwick.ac.uk \\ 3 Bradley Department of Electrical and Computer Engineering, Virginia Tech, Blacksburg, VA 24060, USA; \\ haosong@vt.edu \\ * Correspondence: gaozhiyang@stu.xidian.edu.cn
}

Received: 30 November 2019; Accepted: 9 January 2020; Published: 12 January 2020

\begin{abstract}
Passive localization is an important part of intelligent surveillance in security and emergency applications. Nowadays, Global Navigation Satellite Systems (GNSSs) have been widely deployed. As a result, the satellite signal receiver may receive multiple GPS signals simultaneously, incurring echo signal detection failure. Therefore, in this paper, a passive method leveraging signals from multiple GPS satellites is proposed for moving aerial target detection. In passive detection, the first challenge is the interference caused by multiple GPS signals transmitted upon the same spectrum resources. To address this issue, successive interference cancellation (SIC) is utilized to separate and reconstruct multiple GPS signals on the reference channel. Moreover, on the monitoring channel, direct wave and multi-path interference are eliminated by extensive cancellation algorithm (ECA). After interference from multiple GPS signals is suppressed, the cycle cross ambiguity function (CCAF) of the signal on the monitoring channel is calculated and coordinate transformation method is adopted to map multiple groups of different time delay-Doppler spectrum into the distance-velocity spectrum. The detection statistics are calculated by the superposition of multiple groups of distance-velocity spectrum. Finally, the echo signal is detected based on a properly defined adaptive detection threshold. Simulation results demonstrate the effectiveness of our proposed method. They show that the detection probability of our proposed method can reach $99 \%$, when the echo signal signal-to-noise ratio (SNR) is only $-64 \mathrm{~dB}$. Moreover, our proposed method can achieve $5 \mathrm{~dB}$ improvement over the detection method using a single GPS satellite.
\end{abstract}

Keywords: cyclic cross ambiguity function; data fusion; GPS; multiple satellites collaboration; passive detection

\section{Introduction}

With the development of space technologies, Global Navigation Satellite Systems (GNSSs) have been widely applied in various applications and have been playing an extremely important role in many fields [1-3]. In a GNSS, GNSS navigation satellites need to be widely distributed and used as radiation sources. Among existing GNSSs [4-7], Global Positioning System (GPS) has been broadly recognized and acknowledged as an advanced and mature technology in target detection due to its wider coverage and shorter observation time compared to other GNSSs, such as the Beidou satellite system [8] and Golbal navigation satellite system (GLONASS). Generally, target detection is conducted using a single GPS as the radiation source [9]. However, due to the widely deployed GNSSs, a GPS satellite signal receiver will inevitably receive multiple GPS signals, causing signal contamination and 
the failure of echo signal detection. Hence, instead of relying on only a single GPS satellite radiation source, effective weak echo signal detection methods using multiple GPS satellite radiation sources need to be studied.

Due to the benefits of global coverage, 24-h operation, and easy-to-access signal sources, GPS navigation satellite signals will be employed as third-party illumination sources to study GPS-based external radiation source target detection methods in this paper. For weak echoes of GPS satellites, many researchers have made preliminary explorations [10-14]. These works focus on studying the detection of a single GPS satellite signal. In fact, different GPS satellites in the zenith may share the same frequency bands to send GPS signals. On the other hand, a near-Earth orbit target may be simultaneously covered by multiple satellite beams. As a result, reference signals transmitted on reference channels may be contaminated by other unexpected GPS signals [15], which makes the estimation of reference signals very difficult. In addition, interference caused by other GPS signals may degrade the performance of direct-path interference (DPI)/multi-path interference (MPI) suppression and the subsequent echo detection on an echo channel [16]. For effective echo detection, multiple GPS signals received on the reference channels and the DPI/MPI on monitoring channels should be purified and suppressed.

On the other hand, the received power of GPS signals is very low due to a long-distance propagation $[17,18]$. Furthermore, the signals after the target reflection, also referred to as the target echo, would be even weaker. Even under the condition that the clutter and interference suppression could be realized, the extremely weak GPS echo still needs a long coherent accumulation time to be detected. In order to improve the detection probability of the target echo, the existing methods improved the detection performance by constructing a multi-station joint detection system and merging the detection results of multiple radiation sources [19-21]. Unfortunately, the signal processing methods introduced in these works are not suitable for GPS signals. Therefore, effective use of the received multiple GPS signals to construct a joint detection system with high signal-to-noise ratio (SNR) is an important technical problem that has to be tackled.

The main contributions of this paper can be summarized as follows. A GPS weak echo signal detection method is proposed based on multi-star data fusion. To be specific, under the condition of multiple satellite sources, multiple reference signals will be mixed into a reference channel, resulting in degrading the DPI and MPI suppression effects on a monitoring channel. Therefore, firstly, the proposed method separates and reconstructs multiple GPS reference signals on the reference channel, based on which the Extensive Cancellation Algorithm (ECA) is used to monitor and suppress DPI and MPI. Then, to address the problem of the weak target reflection echo, which is very difficult to be detected, a coordinate conversion algorithm is applied to fuse detection statistics of multiple GPS satellites and obtain a final detection statistic. By this way, the peak value of weak echo detections and the probability of weak echo detections could be improved. Finally, by defining an adaptive detection threshold, the weak echo could be adaptively detected.

The reminder of this paper is organized as follows. In Section 2, the system model under multiple GPS satellite radiation sources is presented. A novel method of joint target detection is proposed and the corresponding technical details are described in the third Section 3, including the separation and reconstruction of direct wave in a reference channel, the suppression of direct and multi-path interference in a monitoring channel, and the construction of detection quantity and the design of detector. In the Section 4, the extensive simulation studies are conducted.

\section{System Model}

The system model of the echo signal detection and reception system based on data fusion of multiple GPS satellites is shown in Figure 1, where $R_{t}$ is the distance from the satellite to a target, $L$ is the distance from a satellite to a receiver, $\theta$ is the arrival angle of the echo, $\varphi$ is the arrival angle of the direct wave, and $R_{r}$ is the distance from a target to a receiver. 


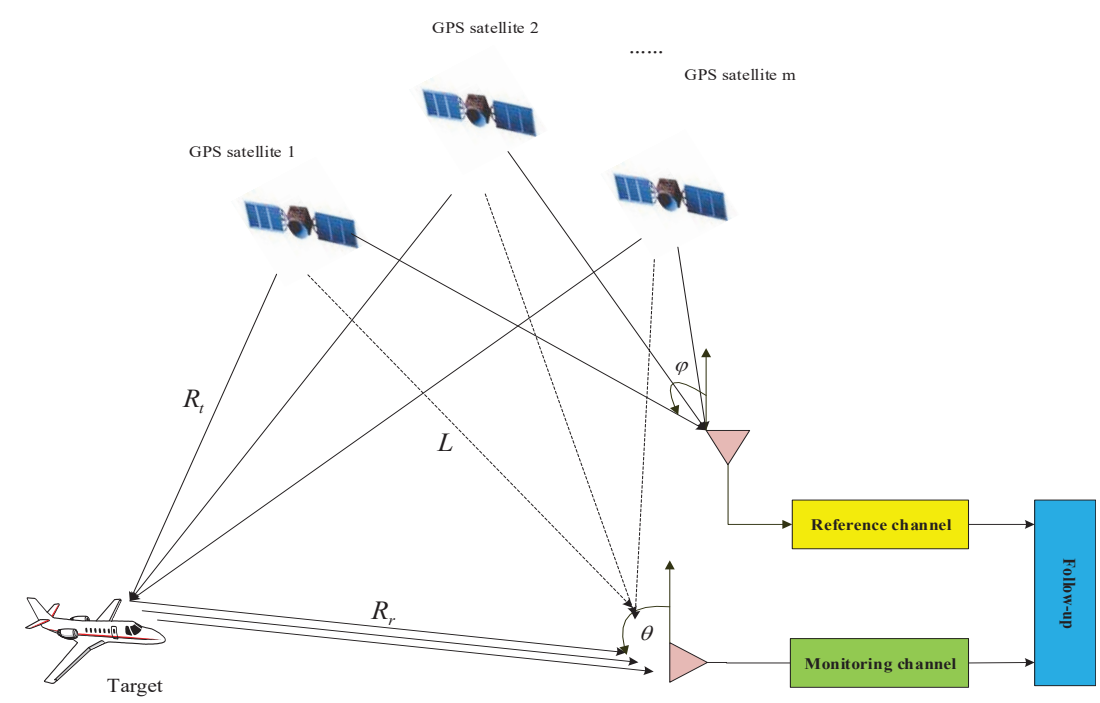

Figure 1. Passive detection based on multiple collaborative GPS satellites.

As shown in Figure 2, a standard GPS receiver exists in the reference channel, which is vertically pointed to the zenith in order to receive the reference signals. Then, the received reference signals will be used for the DPI and MPI suppression in the monitoring channel. Through the monitoring channel, the receiver is able to realize the self-positioning of the detection system, the baseline measurement, and the tracking of the current satellite, obtaining the ephemeris position information of the zenith at the current moment. These measurements will facilitate subsequent offline signal processing.

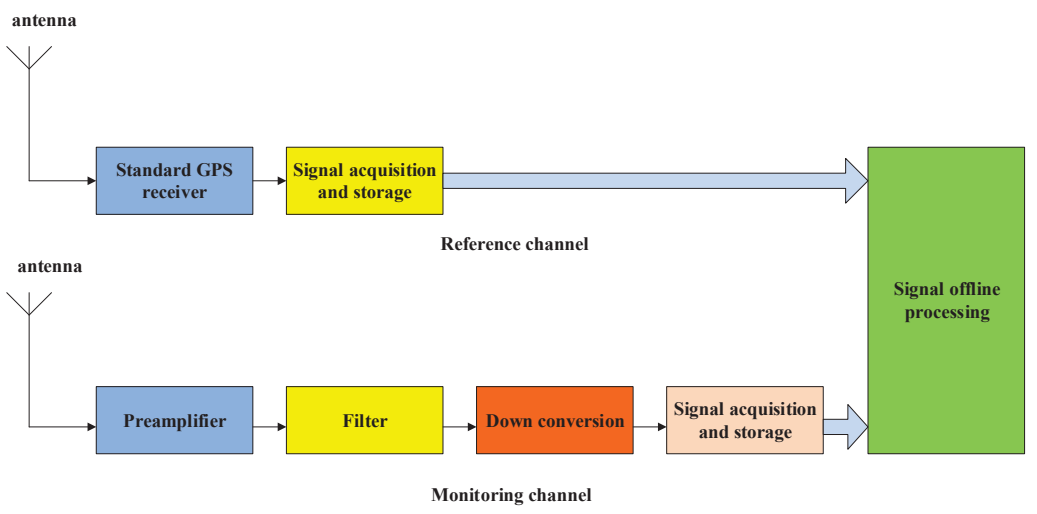

Figure 2. The diagram of the receiving signal channels.

The monitoring channel for reflected echo receptions is mainly composed of a GPS receiving antenna, an amplifier, a filter, a down conversion circuit, and a digital storage oscilloscope. In the GPS receiving antenna, a left-handed circularly polarized receiving antenna is deployed, which is tilted towards the target. The amplifier amplifies the GPS echo signal to achieve a $20 \mathrm{~dB}$ to $30 \mathrm{~dB}$ amplification. The filter is employed for interference cancellation of clutter signals outside the GPS L-band, reducing the influence of out-of-band clutter on subsequent detection processes. The down-conversion module is applied to down-convert the GPS signal from the $L$-band to the intermediate frequency to reduce the complexity of the processing. The digital storage oscilloscope is used for the rapid sampling and storage of data to enable following offline processing.

In the monitoring channel, it is assumed that there are M GPS satellites as the radiation source. In addition to the reflected echoes received by the plurality of GPS satellite signals from the target, on the monitoring channel, direct wave signals and multipath interference can be received, which are 
produced by the reflection of GPS direct wave signals from close ground objects. Accordingly, the received signals of the monitoring channel can be expressed as

$$
\begin{aligned}
x_{s}(t)= & \alpha_{1} x_{1}\left(t-\tau_{1}\right) \exp \left(j 2 \pi f_{d_{1}}\right)+\alpha_{2} x_{2}\left(t-\tau_{2}\right) \exp \left(j 2 \pi f_{d_{2}}\right)+\ldots+\alpha_{M} x_{M}\left(t-\tau_{m}\right) \exp \left(j 2 \pi f_{d_{M}}\right) \\
& +\sum_{i=0}^{W_{1}} \omega_{1_{i}} x_{1}\left(t-\tau_{1_{i}}\right)+\sum_{i=0}^{W_{2}} \omega_{2_{i}} x_{2}\left(t-\tau_{2_{i}}\right)+\ldots \ldots+\sum_{i=0}^{W_{M}} \omega_{M_{i}} x_{M}\left(t-\tau_{M_{i}}\right)+n_{s}(t),
\end{aligned}
$$

where $\alpha_{M} x_{M}\left(t-\tau_{m}\right) \exp \left(j 2 \pi f_{d_{M}}\right)$ represents the echo signal of the Mth GPS satellite, $\alpha_{M}$ represents the amplitude of the echo signal, and $x_{M}(t)$ is the Mth GPS satellite signal. In addition, $\tau_{M}$ and $f_{d_{M}}$ are the delay and frequency offset of that signal, respectively, $n_{s}(t)$ is the noise of the monitoring channel, $\sum_{i=0}^{W_{M}} \omega_{M_{i}} x_{M}\left(t-\tau_{M_{i}}\right)$ stands for the multipath of the Mth GPS satellite affected by multipath interference, $i$ represents the subscript of the $i$ th path in the multipath, $\mathrm{W}_{M}$ is the number of multipath components of the Mth GPS signal, $\omega_{M_{i}}$ is the gain of the $i$ th path in the Mth GPS satellite signal, and $\tau_{M_{i}}$ is the delay of the $i$ th path in the Mth GPS satellite signal.

In the reference channel, GPS satellites share and reuse the same frequency band due to the characteristics of GPS satellite system distribution and Code Division Multiple Access (CDMA) modulation. Thus, the ground receiver is likely to receive more than four frequency-overlapped GPS signals. The received signal by the GPS receiver could be given by

$$
x_{r}(t)=\sum_{k=1}^{M} x_{k}\left(t-\tau_{k}\right)+n_{r}(t)
$$

where $x_{k}\left(t-\tau_{k}\right)$ is the direct wave signal of the $k$ th GPS satellite, and $n_{r}(t)$ represents the noise of the reference channel.

\section{Interference Suppression}

Since GPS signals may be transmitted on the same frequency bands and the zenith can simultaneously have multiple GPS satellites, a near-earth orbit target can be simultaneously illuminated by multiple satellite beams. Therefore, multiple different GPS signals may be received on the reference channel, contaminating desired reference signals and degrading the suppression of DPI and MPI.

\subsection{Influence of Reference Channel Interference on DPI and MPI Suppression}

To suppress DPI and MPI, an adaptive filtering algorithm was adopted [22-24]. The direct wave signal received on the reference channels is used as reference signals to cancel the DPI and MPI on the monitoring channels. The specific suppression principle is shown in Figure 3, where $X_{\text {ref }}(n)$ is the direct wave signal of the reference channel, $X_{s}(n)$ is the mixed signal received by the monitoring channel, and $W(n)$ is the coefficient of the filter. The algorithm is able to adjust the filter coefficients adaptively to minimize the output error $e(n)$ of the filter, and $e(n)$ also gives the signal for the monitoring channel after interference suppression, which is obtained by

$$
e(n)=X_{s}(n)-W^{H}(n-1) X_{r e f}(n) .
$$

From Figure 3, reference signals are required in this method. The reference signal is used to cancel the DPI and MPI in the monitoring channel, and is also used as a reference signal for time-frequency two-dimensional correlation with the echo signal in the monitoring channel. Therefore, the reference signal is very important throughout the process. This section analyzes the influence of reference channel noise and interference signals on the direct wave multipath suppression. In Figure 4, the DPI and MPI suppression are performed by using the algorithm in Figure 3, and the monitoring channel signal and the reference signal after suppression are used as fuzzy functions, and the DPI and MPI 
inhibition effects are judged by observing whether or not there is a peak corresponding to the echo on the time delay-Doppler spectrum.

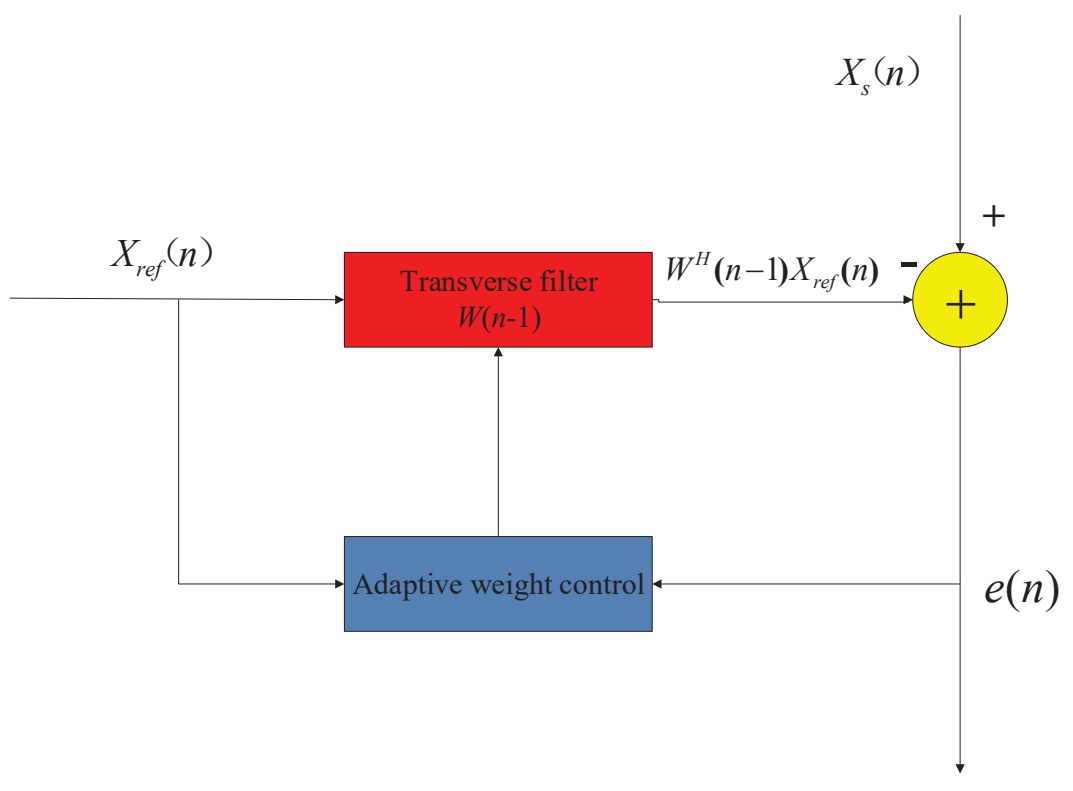

Figure 3. The suppression methods of DPI and MPI.

First, set the monitoring channel to include both the target echo and DPI and MPI. The reference channel only has the reference signal and noise corresponding to the target echo. There are no other GPS interference signals. The parameter settings are shown in Table 1. The DPI and MPI suppression effects are assessed by observing whether there is a peak corresponding to the echo on the delay-Doppler spectrum, which are shown in Figures 4 and 5. As can be seen from Figure 4, when the SNR of the reference signal is $-15 \mathrm{~dB}$, after using this reference signal to perform DPI and MPI suppression on the monitoring channel, the interference peak in the delay-Doppler spectrum is still strong, making the peak corresponding to the target echo invisible. When the SNR of the reference signal is $5 \mathrm{~dB}$, after the suppression of the direct wave, it can be seen from Figure 5 that, although the interference peaks of DPI and MPI still exist, the peak of the echo can be seen in the delay-Doppler spectrum. If the SNR is gradually increased, DPI and MPI can be completely suppressed. Therefore, it can be concluded that the noise of the reference channel has a great influence on the suppression process of DPI and MPI.

Table 1. Parameter setting.

\begin{tabular}{cccc}
\hline & Doppler Shift (Hz) & Delay (us) & Power (dBm) \\
\hline The noise of the reference channel & - & - & $-105 /-95$ \\
The reference signal in the reference channel & 0 & 0 & -110 \\
The target echo of the monitoring channel & 500 & 5 & -150 \\
\hline
\end{tabular}

Second, set the echo channel to include the target echo plus DPI and MPI. In this case, only the reference signal and other GPS interference signals are in the reference channel. The parameter settings are shown in Table 2.

Table 2. Parameter setting.

\begin{tabular}{cccc}
\hline & Doppler Shift $\mathbf{( H z )}$ & Delay (us) & Power (dBm) \\
\hline The reference signal in reference channel & 0 & 0 & -100 \\
The interference signal 1 and 2 in reference channel & 0 & 0 & $-110 /-140$ \\
The noise of reference channel & - & - & - \\
The target echo of monitoring channel & 500 & 5 & -140 \\
\hline
\end{tabular}




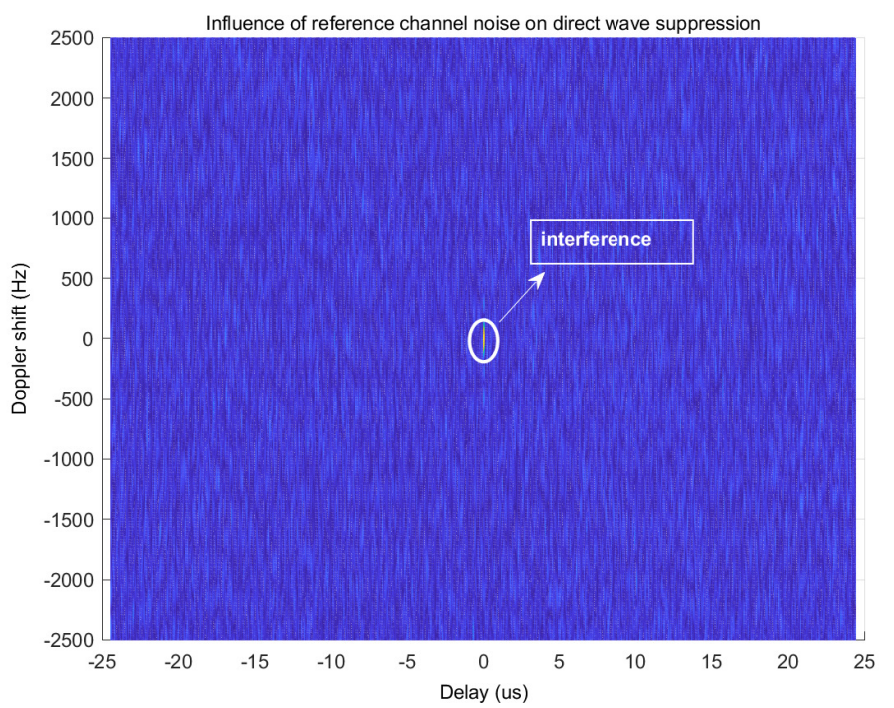

Figure 4. The influence of reference channel SNR on interference suppression process with $\mathrm{SNR}=-15 \mathrm{~dB}$.

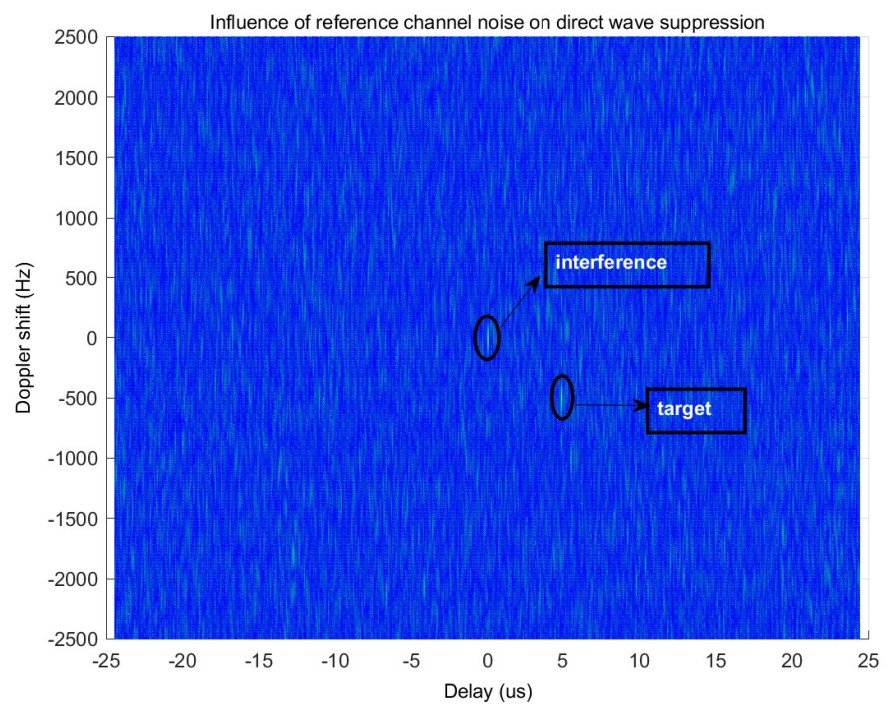

Figure 5. The influence of reference channel SNR on interference suppression process with $\mathrm{SNR}=5 \mathrm{~dB}$.

As can be seen from Figure 6, when the power of the interference signal in the reference channel is $-110 \mathrm{dBm}$, that is, the signal-to-interference ratio (SIR) is $10 \mathrm{~dB}$, the peak corresponding to the target is still invisible after interference suppression, and the DPI and MPI suppression methods fail in this scenario. As can be seen from Figure 7, when the power of the interference signal in the reference channel is reduced to $-140 \mathrm{dBm}$ and the SIR is $30 \mathrm{~dB}$, the peak corresponding to the target can be seen after interference suppression, but the interference is still not eliminated. Therefore, it can be concluded that, under normal circumstances, the reference channel inevitably receives signals from multiple GPS satellites as co-channel interference, and it has a great influence on the suppression process of DPI and MPI. 


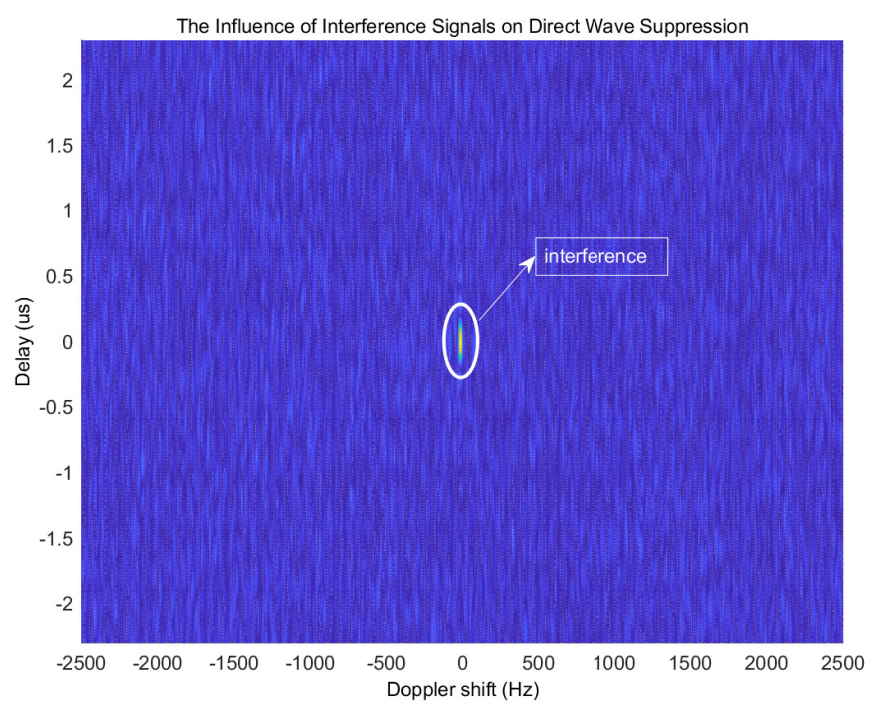

Figure 6. The influence of reference channel SIR on DPI and MPI suppression with SNR $=10 \mathrm{~dB}$.

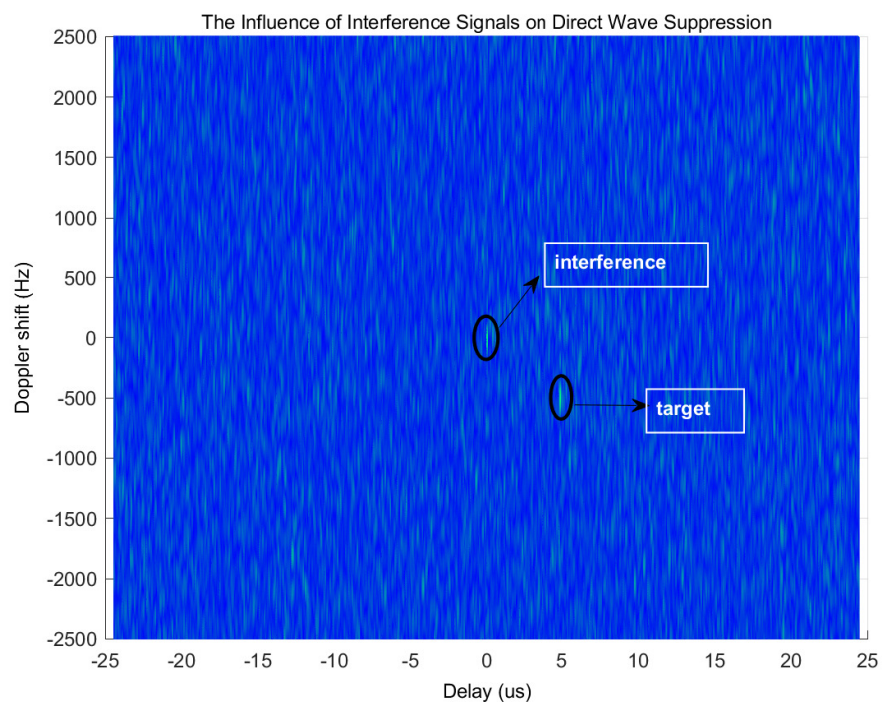

Figure 7. The influence of reference channel SIR on DPI and MPI suppression with $\mathrm{SNR}=30 \mathrm{~dB}$.

Based on the above analysis, the noise and interference signals in the reference channel have a great influence on DPI and MPI suppression. The traditional external source detection system does not process the reference channel signal and uses it directly as a reference signal for the DPI and MPI interference suppression algorithms. In order to correctly detect the echo signal, the GPS reference signal of the reference channel must be purified and separated.

\subsection{Multiple GPS Signals Separation and Reconstruction}

The GPS signal uses C/A code $C(t)$ and $P$ code $P(t)$ to spread the data code $D(t)$. This paper only considers the GPS satellite signal modulated by C/A code [25,26]. The signal received by the reference channel can be expressed as

$$
x_{r}(t)=\sum_{k=1}^{M} \sqrt{P_{k}} \cdot C_{k}\left(t-\tau_{k}\right) \cdot D_{k}\left(t-\tau_{k}\right) \cdot e^{j 2 \cdot \pi k t}+n(t),
$$


where $P_{k}$ is the power of the transmitted signal of the kth GPS satellite, $C_{k}(t)$ represents the C/A code of the kth GPS satellite, $D_{k}(t)$ represents the navigation data of the kth GPS satellite, $f_{k}$ is the carrier frequency of the received signal, and $\tau_{k}$ is the delay of the received signal. By using the CDMA principle of the GPS system and the characteristics of the C/A code disclosure, a successive interference canceller (SIC) can be used to effectively separate and reconstruct multiple GPS signals $x_{r}(t)$ by the reference channel.

SIC is implemented in multiple steps, where each step requires signal acquisition to reconstruct the signal; then, the interference signal is removed from the received signal, and the "detection-reconstruction-cancel" step is repeated until all GPS signals are recovered. The steps of the method are as follows:

Step 1: Using a GPS acquisition algorithm to detect the GPS signal in the received signal of the reference channel, and obtain its corresponding spreading code information $C_{l}(t)$, amplitude estimation value $P_{l}$, phase offset value $\tau_{l}$, and frequency offset value $f_{l}$;

Step 2: Demodulate and reconstruct the signal $x_{l}(t)$; the received signal is down-converted by using the frequency offset information $f_{l}$, and then the phase offset $\tau_{l}$ is obtained by the lth local C/A code $C_{l}(t)$ to obtain $C_{l}\left(t-\tau_{l}\right)$, and, according to the orthogonality of the C/A codes of different satellites, the information $D_{l}^{\prime}(t)$ of the satellite is de-spreaded. In order to correctly recover the navigation data, the de-spreaded data are processed by the envelope averaging method, and finally the navigation data $D_{l}(t)$ are determined. The process is shown in Figure 8.
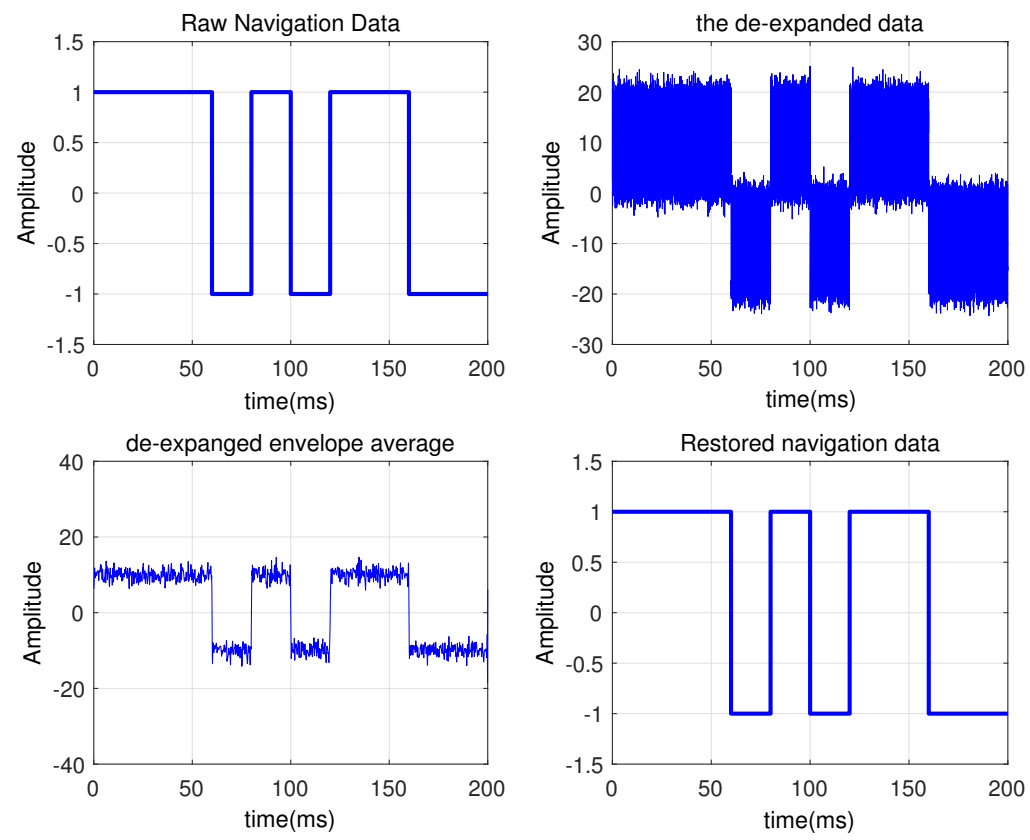

Figure 8. Reference signal demodulation process.

The recovered navigation data $D_{l}(t)$ is modulated using the phase-synchronized local C/A code, and the reconstructed reference signal $x_{l}(t)$ is obtained by using the amplitude $P_{l}$ of the signal and then up-converting, which is expressed as

$$
\widehat{x}_{l}(t)=\sqrt{P_{l}} \cdot C_{l}\left(t-\tau_{l}\right) D_{l}\left(t-\tau_{l}\right) e^{j 2 \pi f_{l} t} .
$$

The correlation coefficient between the original signal and the reconstructed signal obtained by simulation calculation is 0.99 . As shown in Figure 9, it shows that the original signal is well reconstructed. 

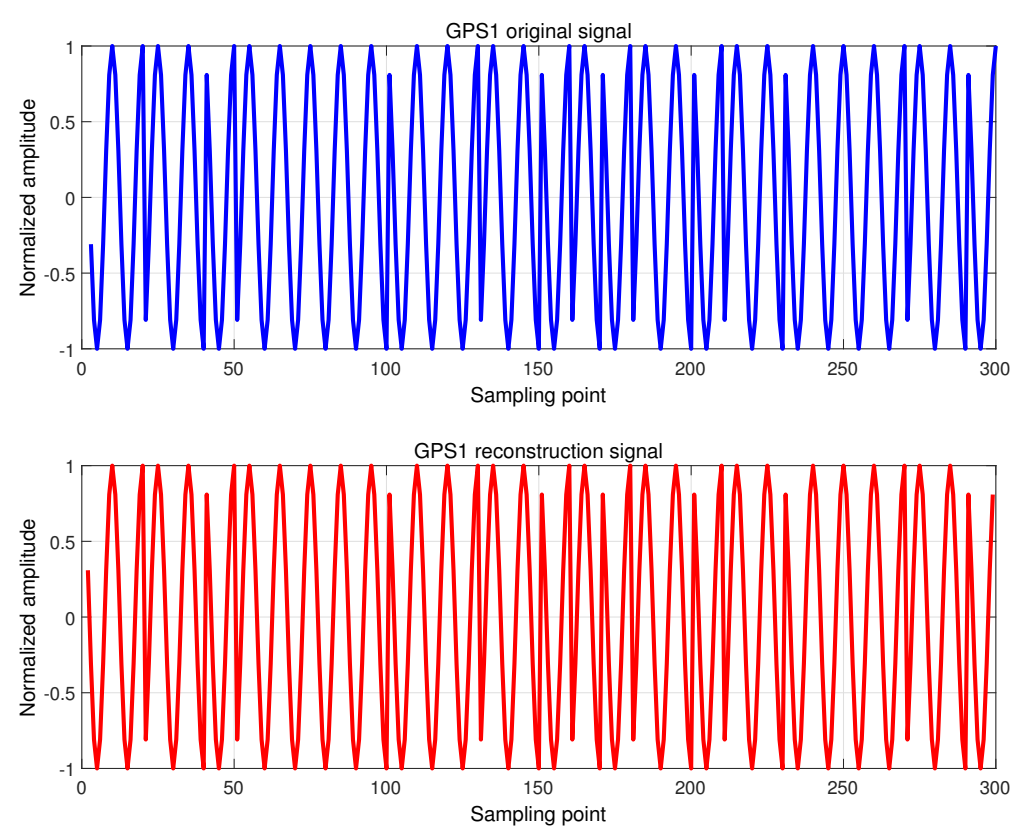

Figure 9. Compare the reconstructed signal with the original signal.

Step 3: Subtract the signal recovered in step 2 from the received signal of the reference channel to reduce the interference when reconstructing the next signal. In this paper, the adaptive filtering method is used to eliminate the interference signal, that is, the input end of the reference signal in Figure 3 is replaced by the reconstructed signal $\widehat{x}_{l}(t)$, and the input end of the monitoring channel signal is replaced by $x_{r}(t)$, so that the signal after strong interference cancellation can be obtained

$$
x_{r}^{(1)}(n)=\varepsilon(n)=x_{r}(n)-w^{T}(n-1) \hat{x}_{l}(n) .
$$

Step 4: Repeat the "capture-reconstruction-cancellation" process from step one to step three for the signal $x_{r}^{(1)}(t)$ of the output signal of step three until the GPS signal is not detected in the reference channel. Finally, a plurality of reconstructed GPS reference signals can be obtained. At this time, the reference signals $\hat{x}_{1}(t), \hat{x}_{2}(t) \ldots \hat{x}_{M}(t)$ have been separated and there is no noise, so there is no other GPS signals and noise interfering with the reference signals. The process not only eliminates the noise in the reference signal, but also separates multiple GPS signals in the reference channel, providing a good reference signal for the DPI and MPI suppression processes of multiple GPS satellites in the monitoring channel. At the same time, it also provides a useful reference signal for the joint detection of multiple GPS weak echoes. In addition, the GPS signal that interferes with the original reference channel is converted into a reference signal that is advantageous to the system.

\subsection{DPI and MPI Suppression Based on ECA}

After the reference signal separation and reconstruction, the DPI and MPI of multiple satellites can be suppressed. Different from the DPI and MPI suppression processes of a single GPS satellite, this section suppresses the DPI and MPI brought by multiple GPSs in the surveillance channel based on the extensive cancellation algorithm (ECA).

Firstly, the ECA uses multiple GPS reference signals $\hat{x}_{1}(t), \hat{x}_{2}(t) \ldots \hat{x}_{M}(t)$ to construct a delay spread matrix $\mathbf{X}^{\text {ref }}$ of multi-satellite signals, which can be expressed as

$$
\mathbf{X}^{\mathrm{ref}}=\left\lfloor\begin{array}{llll}
\mathbf{X}_{1}^{\mathrm{ref}} & \mathbf{X}_{2}^{\mathrm{ref}} & \ldots \mathbf{X}_{i}^{\mathrm{ref}} \ldots & \mathbf{X}_{M}^{\mathrm{ref}}
\end{array}\right\rfloor,
$$


where $\mathbf{X}_{i}^{\text {ref }}(i \in(1, M))$ is the reconstructed matrix of the reconstructed ith reference signal $\hat{x}_{i}(t)$ through different delays, and the extension matrix $\mathbf{X}_{i}^{\text {ref }}$ is an element in the matrix $\mathbf{X}^{\text {ref }}$ and can be expressed as

$$
\mathbf{X}_{i}^{\mathrm{ref}}=\left[\begin{array}{c|c|cc}
\hat{x}_{i}(0) & \hat{x}_{i}(N-1) & \ldots & \hat{x}_{i}(N-K) \\
\hat{x}_{i}(1) & \hat{x}_{i}(0) & \ldots & \hat{x}_{i}(N-K+1) \\
\cdots & \ldots & \ldots & \ldots \\
\hat{x}_{i}(N-1) & \hat{x}_{i}(N-2) & \ldots & \hat{x}_{i}(N-K-1)
\end{array}\right],
$$

where $N$ is the number of sampling points, and $K$ is the maximum delay, which can be obtained by dividing the maximum detection distance by the speed of $\operatorname{light}\left(K=\frac{R_{\max }}{c}\right)$. DPI and MPI can be expressed as

$$
D M P I=\sum_{i=0}^{W_{1}} \omega_{1_{i}} x_{1}\left(t-\tau_{1_{i}}\right)+\sum_{i=0}^{W_{2}} \omega_{2_{i}} x_{2}\left(t-\tau_{2_{i}}\right)+\ldots \ldots .+\sum_{i=0}^{W_{M}} \omega_{M_{i}} x_{M}\left(t-\tau_{M_{i}}\right) .
$$

Then, adjust the value of $\varepsilon=\left[\varepsilon_{0}, \varepsilon_{1} \ldots \varepsilon_{\mathbf{N}-1}\right]^{\mathbf{T}}$ to make $\varepsilon \mathbf{X}^{\text {ref }}$ approach the direct wave and multipath interference. The problem is transformed into the following problem:

$$
\min \left\|x_{S}(t)-\varepsilon \mathbf{X}^{\mathrm{ref}}\right\|^{2}
$$

The solution of Equation (10) uses the least squares criterion [27-29]; then, Equation (10) is equivalent to the following:

$$
\frac{\partial\left(\left\|x_{S}(t)-\varepsilon \mathbf{X}^{\text {ref }}\right\|^{2}\right)}{\partial(\varepsilon)}=0 .
$$

Thus, $\varepsilon=\left(\left(\mathbf{X}^{\mathrm{ref}}\right)^{H} \mathbf{X}^{\mathrm{ref}}\right)^{-1} \mathbf{X}^{\mathrm{ref}} x_{s}(t)$ is obtained, where $\left(\mathbf{X}^{\mathrm{ref}}\right)^{H}$ is the transpose of $\mathbf{X}^{\mathrm{ref}}$, and the signal in the monitoring channel after interference suppression is expressed as

$$
x_{s}^{\prime}(t)=x_{s}(t)-D M P I=x_{s}(t)-\varepsilon \mathbf{X}^{\mathrm{ref}}=x_{s}(t)-\mathbf{X}^{\mathrm{ref}}\left(\left(\mathbf{X}^{\mathrm{ref}}\right)^{H} \mathbf{X}^{\mathrm{ref}}\right)^{-1} \mathbf{X}^{\mathrm{ref}} x_{S}(t),
$$

where $x_{s}^{\prime}(t)$ only contains the echo signal and noise $n_{s}(t)$.

The proposed algorithm does not need to know the gain value, and the DMPI can be directly solved by the proposed algorithm. In order to verify the DPI and MPI suppression algorithms based on signal separation and reconstruction proposed in this paper, the specific parameter settings are shown in Table 3 as follows: the reference channel contains noise, five GPS signals; the monitoring channel contains noise, three GPS echoes, and DPIs corresponding to five reference channel GPS signals. Firstly, the reference signal separation and reconstruction algorithms are used to separate and reconstruct the reference signals $1,2,3,4$, and 5 of the reference channel. Then, the purified reference signal is used together with the suppression algorithm proposed in this section to perform DPI suppression on the signal of the monitoring channel. Finally, the signal of the monitoring channel after the suppression and the reference signal are subjected to cross ambiguity function (CAF) processing [30-32], and the DPI suppression effect is judged by observing whether there is a peak corresponding to the echo on the delay-Doppler spectrum.

Figure 10 shows the time-frequency two-dimensional correlation of un-suppressed DPI of multiple GPS satellites. It can be seen that the echo generated by the target is completely submerged in the peak generated by DPI. Figure 11 shows the comparison of the monitoring channel signals before and after the DPI and MPI suppression methods proposed in this paper. It can be seen that the amplitude of the monitoring channel signal decreases after interference suppression, which proves that strong DPI interference has been effectively suppressed. 
Table 3. Parameter setting.

\begin{tabular}{cccc}
\hline & Doppler Shift (Hz) & Delay (us) & Power (dBm) \\
\hline The noise of reference channel & - & - & -95 \\
Reference channels reference signal $(1,2,3,4,5)$ & 0 & $0 / 2 / 3 / 5 / 6$ & $-100 /-105 /-110 /-112 /-106$ \\
The noise of monitoring channel & - & - & -110 \\
Monitoring channel's target echo $(1,2,3)$ & $500 / 650 / 850$ & $5 / 5.2 / 10$ & $-140 /-145 /-150$ \\
Monitoring channel DPI & 0 & $0 / 2 / 3 / 5 / 6$ & $-100 /-105 /-110 /-112 /-106$ \\
\hline
\end{tabular}

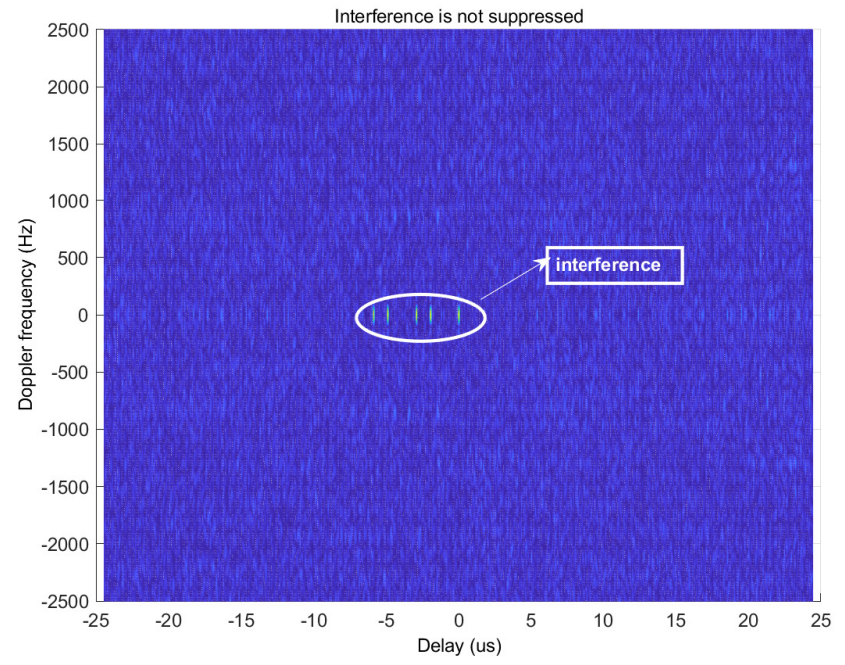

Figure 10. Time-frequency two-dimensional correlation graph with unsuppressed interference.
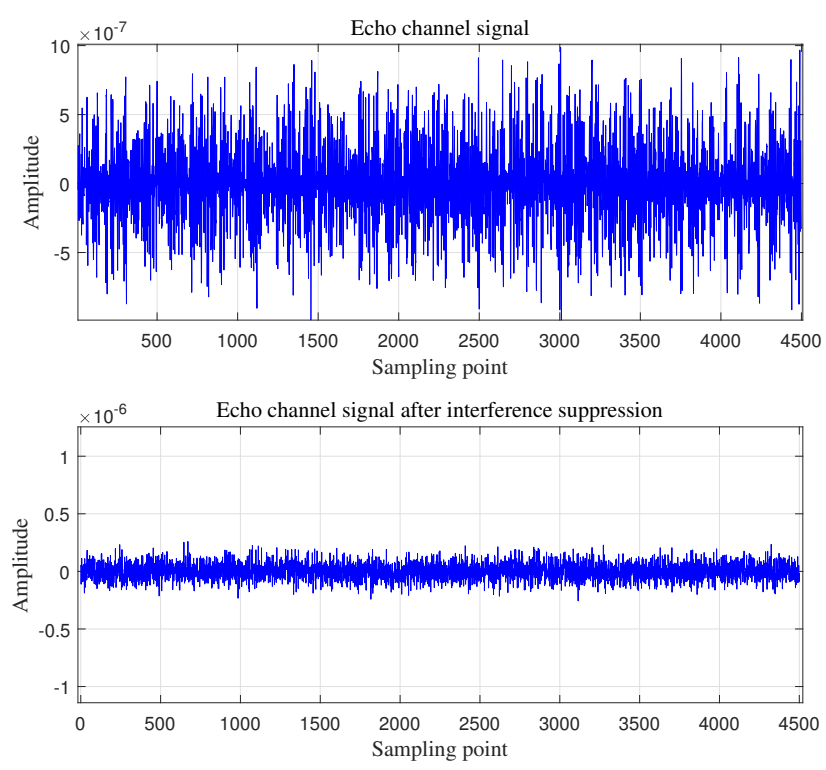

Figure 11. Monitoring channel signal before and after interference suppression.

Figure 12 shows the CCF of the reference and echo signals after DPI interference suppression. It can be seen that the peak of the echo signal is clearly highlighted after the DPI interference suppression. Figure 12 proves that the interference suppression scheme proposed in this paper can effectively suppress the DPI of the monitoring channel when the reference channel SNR is as low as $-15 \mathrm{~dB}$, and the reference channel has multiple GPS signals with similar power. 


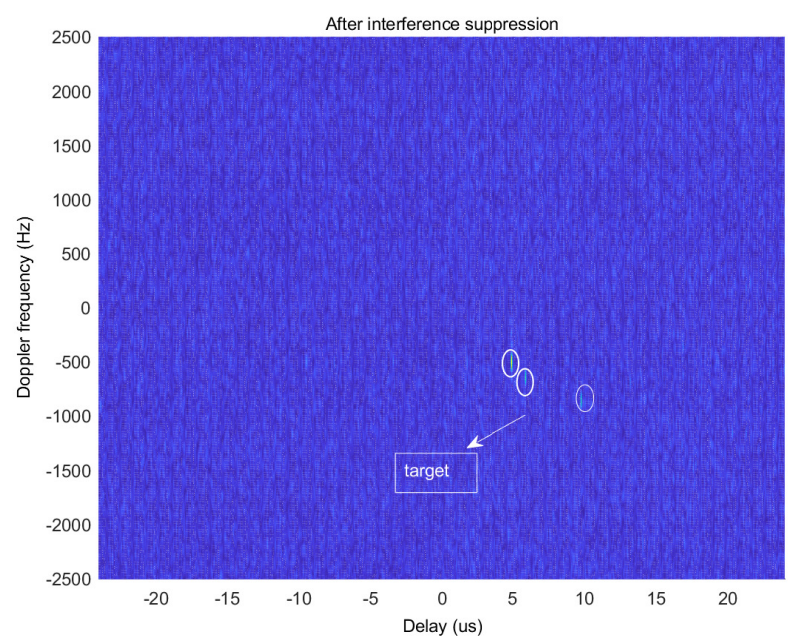

Figure 12. Time-frequency two-dimensional graph after direct wave multipath interference suppression.

This section uses the multiple GPS reference signals separated and reconstructed to construct the delay spread matrix $\mathbf{X}^{\text {ref }}$ of the multiple satellite signals, and then finds the optimal weight $\varepsilon$ based on the least squares criterion. Then, let $\varepsilon \mathbf{X}^{\text {ref }}$ approach multipath and then subtract $\varepsilon \mathbf{X}^{\text {ref }}$ from the monitoring channel to get the monitoring channel signal after DPI and MPI suppression.

\section{Detection Statistics Construction}

Due to the characteristics of the external radiation source detection system and the weak power of the GPS itself, the target echo power is relatively low. The traditional CAF often needs to increase the coherence time of the direct wave and the echo to accumulate the energy of the weak echo [33]. However, long-term coherent accumulation leads to an increase in computational complexity, and, since the range of detection is limited and the target moves faster, the accumulation time is greatly limited. In order to effectively enhance the signal-to-noise ratio of the detected quantity under the condition of equivalent coherence time, in this section, the anti-jamming properties of cycle cross ambiguity function (CCAF) are used to construct the detection statistics of multiple GPS weak echoes, and the coordinate detection algorithm is used to fuse multiple detection statistics to obtain the final detection statistics. This process enhances the signal-to-noise ratio of the detection statistic from the two aspects of detection structure and multi-star data fusion, and improves the detection probability of weak echo. In order to judge the performance of CCAF in the multi-satellite system compared with the traditional CAF detection structure, in this paper, the CAF-based detection statistic and the CCAF-based detection metric are constructed respectively, and the theoretical analysis and simulation performance verification are carried out, respectively.

\subsection{Detection Statistic Construction with a Single GPS Satellite}

\section{(1) Detection statistics based on CAF}

The signal $\hat{x}_{i}(t)$ of the reconstructed reference signal $\hat{x}_{1}(t), \hat{x}_{2}(t) \ldots \hat{x}_{M}(t)$ is selected, and the carrier frequency information $f_{i}$ of the locally known reference signal $\widehat{x}_{i}(t)$ is used for down-conversion processing to obtain the down-converted reference signal $\hat{x}_{i}^{I F}(t)$. Then, the same down-conversion process is performed on the monitoring channel signal $x_{s}^{\prime}(t)$ after DPI and MPI suppression, and the down-converted reference signal $x_{s}^{I F}(t)$ is obtained. In addition, calculate the CAF of $\hat{x}_{i}^{I F}(t)$ and $x_{s}^{I F}(t)$ to obtain the Doppler-time delay spectrum of the i-th GPS satellite, which is expressed as

$$
S_{i}(\tau, f)=\int_{-T / 2}^{T / 2} \hat{x}_{i}^{I F}(t) x_{s}^{I F}(t-\tau) e^{j 2 \pi f t} d t
$$


The discretization of Equation (13) is expressed as

$$
S_{i}(\tau, f)=\sum_{n=0}^{N-1} \hat{x}_{i}^{I F}\left(n T_{s}\right) x_{s}^{I F}\left(n T_{s}-\tau\right) e^{j 2 \pi f n T_{s}}
$$

where $\tau$ is the delay, $f$ is the Doppler shift, $T$ represents the accumulation time, $T_{S}$ stands for the sampling period, and $N$ is the number of sampling points.

(2) Detection statistics based on CCAF

To construct a detection using CCAF, we first need to do a cyclic autocorrelation of the reference signal $\hat{x}_{i}^{I F}\left(n T_{s}\right)$ as

$$
R_{r_{i} r_{i}}^{\alpha}(\tau)=\frac{1}{N} \sum_{n=0}^{N-1} \hat{x}_{i}^{I F}\left(n T_{s}+\tau / 2\right) \hat{x}_{i}^{I F}\left(n T_{s}-\tau / 2\right)^{*} e^{-j 2 \pi \alpha n T_{s}} .
$$

Then, the cyclic cross-correlation of the reference signal $\hat{x}_{i}^{I F}\left(n T_{s}\right)$ and the echo signal $\hat{x}_{s}^{I F}\left(n T_{s}\right)$ is

$$
R_{r, s}^{\alpha-f}(\tau)=\frac{1}{N} \sum_{n=0}^{N-1} x_{s}^{I F}\left(n T_{s}+\tau / 2\right) \hat{x}_{i}^{I F}\left(n T_{s}-\tau / 2\right)^{*} e^{-j 2 \pi \alpha n T_{s}}
$$

where $\tau$ is the delay, $\alpha$ represents the cyclic frequency, and $N$ stands for the number of sampling points.

The vectors at the cyclic frequencies $\alpha^{\prime}$ and $\alpha^{\prime}-f^{\prime}$ corresponding to the maximum peak values of $R_{r_{i} r_{i}}^{\alpha}(\tau)$ and $R_{r_{i} s}^{\alpha}(\tau)$ are respectively extracted, and are recorded as $R_{r_{i} r_{i}}^{\alpha^{\prime}}(\tau)$ and $R_{r_{i} s}^{\alpha^{\prime}-f^{\prime}}(\tau)$. The mutual fuzzy function processing is performed on these two vectors to obtain

$$
\Psi_{i}(u, f)=\sum_{\tau=0}^{N-1} R_{r_{i} s}^{\alpha^{\prime}-f^{\prime}}(\tau) R_{r_{i} r_{i}}^{\alpha^{\prime}}(\tau-u)^{*} e^{j \pi f \tau}
$$

where $\Psi_{i}(u, f)$ represents the CCAF between the monitoring channel signal and the reference signal, $u$ is the delay, and $f$ is the Doppler shift.

\subsection{Detection Statistics Construction with Multiple GPS Satellites}

Due to the different distribution positions of different GPS satellites, the peak coordinates of multiple GPS satellite echo detections $S_{i}(\tau, f)$ and $\Psi_{i}(u, f)$ are also different. Thus, it is impossible to add a plurality of detection amounts to the fusion structure detection statistic. Aiming at this problem, this section unifies the detection peak coordinates of different satellites by coordinate transformation, which can superimpose the echo detection spectrum of several different GPS satellites to achieve the purpose of non-correlated cumulative enhanced signal-to-noise ratio. Thereby, a detection statistic with a higher SNR is constructed.

Figure 13 shows the geometry of the receiving system, where $\theta$ is the signal arrival angle, $\delta$ is the angle between the bistatic angle bisector and the speed $v$ of the aircraft, and $\beta$ is the bistatic angle. It can be seen from the figure that the positions of different GPS satellites are different, so the delay $\tau$ and the Doppler shift $f_{d}$ corresponding to the peak values of the two-dimensional correlation between the different satellite reference signals and the monitoring channel echo signals are different. However, the common edge $R_{r}$ and the velocity $v$ corresponding to different peak coordinates $\tau$ and $f_{d}$ are the same, so the detection spectrum can be converted from the delay-Doppler dimension to the distance velocity dimension so that the coordinate peaks are the same. Thereby, it is possible to accumulate different detection amounts. The relationship between $\tau$ and $R_{r}$ and $f_{d}$ and $v$ is

$$
\left\{\begin{array}{c}
R_{r}+R_{t}=L+c \tau \\
R_{t}^{2}=R_{r}^{2}+L^{2}-2 R_{r} L \cos \theta
\end{array}\right.
$$


Solving Equation (18) can obtain:

$$
\begin{gathered}
R_{r}=\frac{c^{2} \tau^{2}+2 L c \tau}{2(L+c \tau-L \cos \theta)}=f(\tau), \\
f_{d}=\frac{2 v}{\lambda} \cos \delta \cos \frac{\beta}{2}=\frac{2 v^{\prime}}{\lambda} \cos \frac{\beta}{2}=g(v),
\end{gathered}
$$

where $v^{\prime}=v \cos \delta$, and $v^{\prime}$ represents the speed $v$ of the target on the bisecting angle bisector, and $\beta$ can be obtained from

$$
\sin (\beta)=\frac{2 R_{r} \sin \theta\left(R_{r}+c \tau-R_{r} \cos \theta\right)}{R_{r}^{2}-2 R_{r}\left(R_{r}+c \tau\right) \cos R_{r}+(L+c \tau)^{2}} .
$$

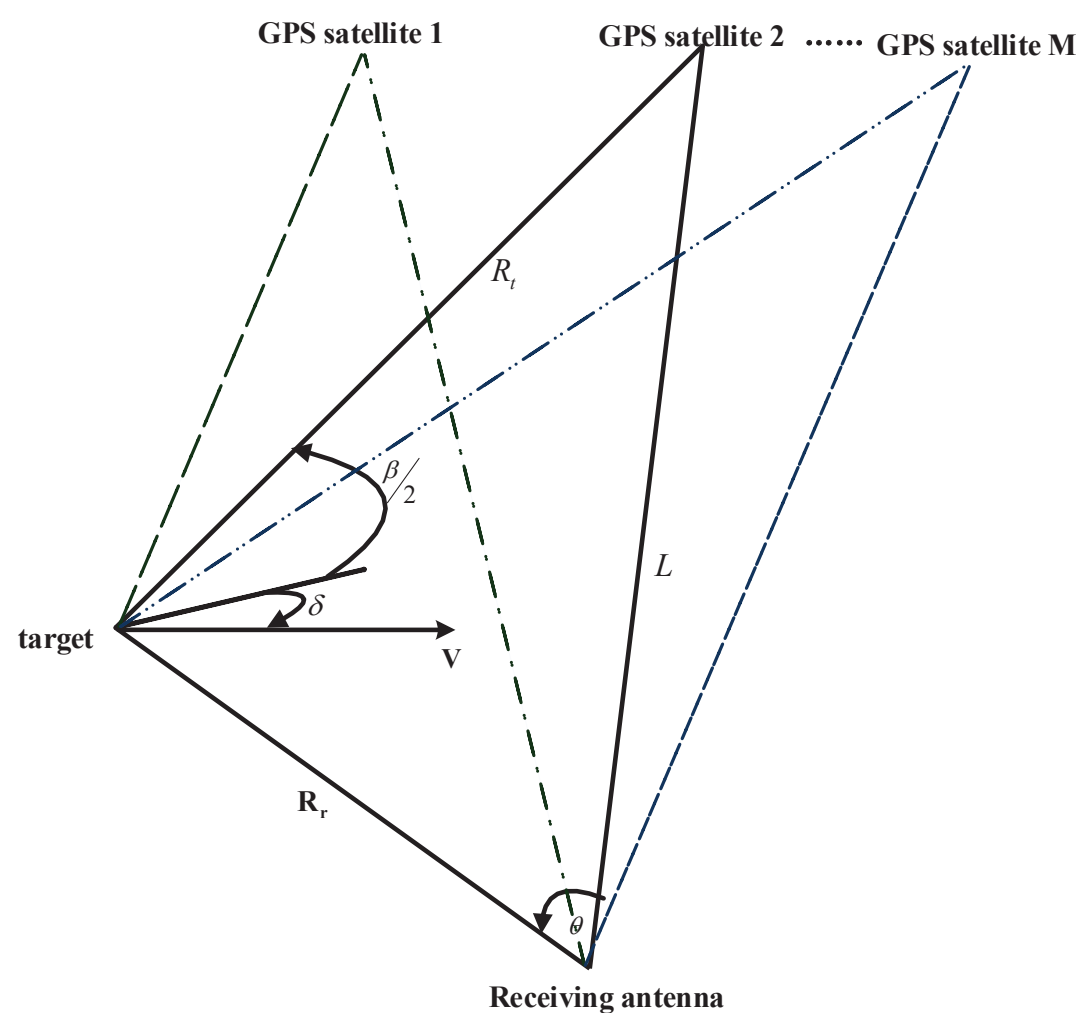

Figure 13. The geometry of the receiving system.

The detection quantities $S_{i}(\tau, f)$ and $\Psi_{i}(u, f)$ obtained by different methods are transformed by Equations (19) and (21) to obtain distance-velocity spectra $S_{i}\left(f^{-1}\left(R_{r}\right), g(v)\right)$ and $\Psi_{i}\left(f^{-1}\left(R_{r}\right), g(v)\right)$, which are expressed as:

$$
\begin{gathered}
S_{i}\left(f^{-1}\left(R_{r}\right), g(v)\right)=\sum_{n=0}^{N} x_{s}^{I F}\left(n T_{s}\right) \hat{x}_{i}^{I F}\left(n T_{s}-f^{-1}\left(R_{r}\right)\right)^{*} e^{j 2 \pi g(v) n T_{s}}, \\
\Psi_{i}\left(f^{-1}\left(R_{r}\right), g(v)\right)=\sum_{n=0}^{N} R_{r_{i} s}^{\alpha^{\prime}-f^{\prime}}\left(n T_{s}\right) R_{r_{i} r_{i}}^{\alpha^{\prime}}\left(n T_{s}-f^{-1}\left(R_{r}\right)\right)^{*} e^{j 2 \pi g(v) n T_{s}} .
\end{gathered}
$$

At this time, the detection amount $S_{i}\left(f^{-1}\left(R_{r}\right), g(v)\right)$ or $\Psi_{i}\left(f^{-1}\left(R_{r}\right), g(v)\right)$ of the plurality of GPS satellites can be non-coherently superimposed in the distance-speed domain. The final superimposed detection statistics obtained by the two methods as

$$
\Lambda\left(R_{r}, V\right)=\sum_{i=1}^{M} S_{i}\left(f^{-1}\left(R_{r}\right), g(v)\right),
$$




$$
\Omega\left(R_{r}, V\right)=\sum_{i=1}^{M} \Psi_{i}\left(f^{-1}\left(R_{r}\right), g(v)\right),
$$

where $R_{r}$ represents the distance from the target to the receiver and $V$ represents the speed of the target. It can be seen from the above process that this paper uses the multiple GPS reference signals separated and reconstructed to calculate the detection quantity of different GPS satellites as the illumination source, which provides more favorable information for target detection. However, due to the different peak values of the detected quantities obtained by multiple GPSs, these detection quantities cannot be effectively fused, so this paper uses the coordinate fusion algorithm to combine them to obtain the final detection amount after peak enhancement.

\section{Moving Aerial Target Detection Performance Analysis}

In order to better design the decision threshold of the detector and evaluate the detection performance of the two detection quantities, this section analyzes the probability distributions of $\Lambda(L, V)$ and $\Omega(L, V)$, respectively.

For convenience, the reference baseband signal $\hat{x}_{i}^{I F}(t)$ of the GPS satellite is first discretized and expressed as

$$
\hat{x}_{i}^{I F}\left(n T_{s}\right)=\beta_{i} p_{i}\left(n T_{s}\right),
$$

where $\beta_{i}$ is the amplitude of the baseband reference signal, $p_{i}\left(n T_{s}\right)$ is the baseband reference signal after the amplitude normalized, and $T_{S}$ stands for the sampling period.

The signal $x_{s}^{I F}(t)$ of the monitoring channel is discretized and then expressed as

$$
x_{s}^{I F}\left(n T_{s}\right)=\sum_{j=1}^{M} \alpha_{j} p_{j}\left(n T_{s}-\tau_{j}\right) \exp \left(j 2 \pi f_{d_{j}} n T_{s}\right)+\omega\left(n T_{s}\right),
$$

where $\alpha_{j}$ is the amplitude of the echo signal, $p_{j}\left(n T_{s}-\tau_{j}\right) \exp \left(j 2 \pi f_{d_{j}} n T_{s}\right)$ is the amplitude-normalized echo signal, $\omega(n)$ is the complex Gaussian noise obeying the $N\left(0, \sigma_{w}^{2}\right)$ distribution, and $N(\cdot)$ is the Gaussian distribution.

The binary hypothesis of echo signal detection is: assuming that $H_{1}$ is the target existence, the signal $x_{s}^{\prime \prime \prime}\left(n T_{s}\right)$ of the monitoring channel contains the echo signal $\alpha_{i} p_{i}\left(n T_{s}-\tau_{i}\right) \exp \left(j 2 \pi f_{d_{i}} n T_{s}\right)$ corresponding to a certain reference signal $\hat{x}_{i}^{I F}\left(n T_{s}\right)$. Assuming that $H_{0}$ indicates that the target does not exist, the signal $x_{s}^{\prime \prime \prime}\left(n T_{s}\right)$ of the monitoring channel does not contain any echo signal corresponding to $\hat{x}_{i}^{I F}\left(n T_{s}\right)$, which is expressed as follows:

$$
\left\{\begin{array}{c}
H_{0}: x_{s}^{I F}\left(n T_{s}\right)=\omega\left(n T_{s}\right) \\
H_{1}: x_{s}^{I F}\left(n T_{s}\right)=\sum_{\substack{j=1 \\
j j=i}}^{M} \alpha_{j} p_{j}\left(n T_{s}-\tau_{j}\right) \exp \left(j 2 \pi f_{d_{i}} n T_{s}\right)+\omega\left(n T_{s}\right) .
\end{array}\right.
$$

\subsection{Performance Analysis of Detection Based on CAF}

Lemma 1. The distribution of the detection statistic under the $H_{1}$ hypothesis is

$$
\left(S_{i}(\tau, f) \mid H_{1}\right) \sim C N\left(\left\{\alpha_{i}^{*} \beta_{i} \chi_{p p}\left(\tau-n_{\tau}, f-f_{d}\right)\right\}, \quad 2 N \beta_{i}^{2} \sigma_{\omega}^{2}\right),
$$

where $C N($.$) represents the complex Gaussian process.$

Proof. See Appendix A.1. 
As with the analysis under the $H_{1}$ hypothesis, it can be concluded that the detection statistic distribution under the $H_{0}$ hypothesis is

$$
\left(S_{i}(\tau, f) \mid H_{0}\right) \sim C N\left(0,2 N \beta^{2} \sigma_{\omega}^{2}\right) .
$$

Using the data fusion technique of multiple GPS satellites, according to the cumulative nature of the Gaussian distribution, the distribution of the final detection statistic $\Lambda\left(R_{r}, V\right)$ is

$$
\begin{gathered}
\left(\Lambda \mid H_{0}\right) \sim C N\left(0, \sum_{i=1}^{M} 2 N \beta_{i}^{2} \sigma_{\omega}^{2}\right), \\
\left(\Lambda \mid H_{1}\right) \sim C N\left(\sum_{i=1}^{M}\left\{\alpha_{i}^{*} \beta_{i} \chi_{p p}\left(\tau-n_{\tau}, f-f_{d}\right)\right\}, \sum_{i=1}^{M} 2 N \beta_{i}^{2} \sigma_{\omega}^{2}\right) .
\end{gathered}
$$

The false alarm probability of detecting weak echo signals from different GPS satellite sources by Equations (28) and (31) is given by

$$
P_{F A}=\int_{\lambda}^{\infty} f\left(\Lambda \mid H_{0}\right) d \Lambda=\exp \left(-\frac{\lambda}{\sum_{i=1}^{M} 2 N \beta_{i}^{2} \sigma_{\omega}^{2}}\right),
$$

where $f\left(\Lambda \mid H_{0}\right)$ is the probability density function of $\left(\Lambda \mid H_{0}\right)$.

From Equation (33), it can be concluded that the adaptive detection threshold $\lambda$ is

$$
\lambda=-\sum_{i=1}^{M} 2 N \beta_{i}^{2} \sigma_{\omega}^{2} \ln \left(P_{F A}\right)
$$

According to Equationss (A2), (32), and (34), the detection probability can be obtained by using signal detection theory:

$$
P_{D}=\int_{\lambda}^{\infty} f\left(\Lambda \mid H_{1}\right) d \Lambda=Q_{m}\left(\sqrt{\frac{\left|\sum_{i=1}^{M}\left(\beta_{i} \alpha_{i} N\right)\right|^{2}}{\sum_{i=1}^{M} N 2 \beta_{i}^{2} \sigma_{\omega}^{2}}}, \sqrt{\frac{\lambda}{\sum_{i=1}^{M} N 2 \beta_{i}^{2} \sigma_{\omega}^{2}}}\right),
$$

where $Q_{m}(\cdot, \cdot)$ is the Marcum $Q$ function, and $f\left(\Lambda \mid H_{1}\right)$ is the probability density function of $\left(\Lambda \mid H_{1}\right)$.

From Equation (35), it can be seen that the theoretical detection probability of the multi-star weak echo joint detection based on the CCA detection quantity construction method is related to the parameters such as the monitoring channel noise, the number of sampling points $N$, the number of satellites, and the false alarm probability. It can be seen that the detection probability is proportional to the number of satellites $M$, that is, the detection probability increases with the number of satellites. It is theoretically proved that the weak echo combined detection of multiple GPS satellites has a higher detection probability than the weak echo detection of a single GPS satellite, but the method does not reflect the noise suppression performance.

\subsection{Performance Analysis of Detection Based on CCAF}

Lemma 2. The probability distribution of $\Psi_{i}(u, f)$ under $H_{1}$ hypothesis is

$$
\Psi_{i}\left((u, f) \mid H_{1}\right) \sim C N\left(\Psi_{R_{i} R_{i}}(u, f), \frac{\sigma_{\omega}^{2} \beta_{i}^{6}}{N}\right)
$$

Proof. See Appendix A.2. 
Using the same analysis method to analyze the $H_{0}$ hypothesis, the distribution of the detection quantity $\Psi_{i}(u, f)$ of a single GPS satellite under the $H_{0}$ hypothesis is given by

$$
\Psi_{i}\left((u, f) \mid H_{0}\right) \sim C N\left(0, \frac{\sigma_{\omega}^{2} \beta_{i}^{6}}{N}\right) .
$$

Using the data fusion technique of multiple GPS satellites, according to the cumulative nature of the Gaussian distribution, the distribution of the final detection statistic $\Omega\left(R_{r}, V\right)$ is

$$
\left(\Omega\left(R_{r}, V\right) \mid H_{0}\right) \sim C N\left(0, \sum_{i=1}^{M} \frac{\sigma_{\omega}^{2} \beta_{i}^{6}}{N}\right)
$$

and

$$
\left(\Omega\left(R_{r}, V\right) \mid H_{1}\right) \sim C N\left(\sum_{i=1}^{M}\left\{\Psi_{R, R_{i}}(u, f)\right\}, \sum_{i=1}^{M} \frac{\sigma_{\omega}^{2} \beta_{i}^{6}}{N}\right) .
$$

According to Equation (38), the false alarm probability can finally obtain:

$$
P_{F A}=\int_{\lambda}^{\infty} f\left(\Omega \mid H_{0}\right) d \Omega=\exp \left(-\frac{\lambda}{\sum_{i=1}^{M} \frac{\sigma_{\omega}^{2} \beta_{i}^{6}}{N}}\right),
$$

where $f\left(\Omega \mid H_{0}\right)$ is the probability density function of $\left(\Omega \mid H_{0}\right)$.

The detection threshold $\lambda$ in the solution Equation (40) is

$$
\lambda=-\ln \left(P_{F A}\right) \cdot \sum_{i=1}^{M} \frac{\sigma_{\omega}^{2} \beta_{i}^{6}}{N} .
$$

According to Equations (A14), (39), and (41), the detection probability can be obtained by using the signal detection theory

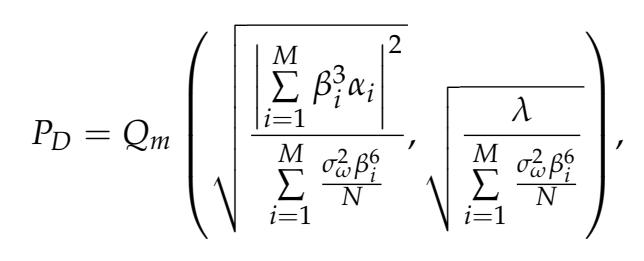

where $Q_{m}(\cdot, \cdot)$ is the Marcum $Q$ function.

It can be seen from Equation (42) that the theoretical detection probability of the GPS weak echo signal detection method based on multi-star data fusion is related to the parameters, such as the monitoring channel noise, the number of sampling points $N$, the number of satellites, and the false alarm probability. It can be seen that the detection probability is proportional to the number of sampling points $N$ and the number of satellites, that is, the detection probability increases with the number of sampling points $N$ and the number of satellites. It is theoretically proved that the GPS weak echo signal detection method based on multi-star data fusion can improve the detection performance from the number of sampling points and the number of satellites. In addition, by comparing Equation (32) and Equation (39), it can be found that the power of the noise of the detection statistic constructed based on the CCAF algorithm decreases as the number of sampling points increases. However, the detection statistic based on the CAF algorithm does not have this property, so the CCAF-based detection statistic construction method has good noise immunity in the proposed algorithm. 


\section{Numerical Results and Discussion}

In order to analyze and verify the effectiveness of the proposed detection algorithm and the influence of various factors on detection, this section presents several simulation experiments using MATLAB (9.5.0.944444 (R2018b), MathWorks Company, Natick, MA, USA) and simulation parameter setting according to $[34,35]$.

Experiment 1: In order to compare the detection performance of CAF and CCAF, this experiment fixes the false alarm probability, the power difference between the reference signal and the echo signal, the number of sampling points, the number of satellites, etc. The delay and Doppler shift of the echo relative to the direct wave are set as 1 us and $500 \mathrm{~Hz}$, respectively. The SNR of the echo is in the range from $-90 \mathrm{~dB}$ to $-30 \mathrm{~dB}$. For different detection quantity construction methods, 2000 Monte Carlo simulation experiments are carried out on the GPS weak echo detection method based on multiple satellites data fusion. The parameters are substituted into the theoretical detection probability Equations (35) and (42) to compare them with the simulation. The specific simulation parameters are shown in Table 4.

Table 4. Parameter setting of experiment 1.

\begin{tabular}{|c|c|c|c|c|c|c|}
\hline Sampling Frequency $(\mathrm{Hz})$ & Duration (ms) & False Alarm Probability & Number of Satellites & Direct Wave Power (dBm) & Echo Power (dBm) & SNR (dB) \\
\hline $10.23 \mathrm{MHz}$ & $1000 \mathrm{~ms}$ & 0.0001 & 1 & -99.3 & -170 & $-90 \sim-30$ \\
\hline
\end{tabular}

It can be seen from Figure 14 that, under the same conditions, the simulated detection probability could reach $99 \%$ at a SNR of $-55 \mathrm{~dB}$. When the SNR of the CCAF algorithm is $-64 \mathrm{~dB}$, the simulated detection probability also reaches $99 \%$. Therefore, the CCAF algorithm is about $9 \mathrm{~dB}$ better than the $\mathrm{CAF}$ algorithm, and the difference between simulation results and theoretical results is $2 \mathrm{~dB}$, which verifies the effectiveness of the method. The above simulation results show that CCAF has a certain noise suppression capability compared with CAF because the noise does not have cyclostationarity. Moreover, according to Equation (A19), it can be seen that the noise power in the CCAF-based detection decreases as the number of points increases, while CAF does not have this property. This experiment verifies the validity of the theory, showing the noise resistance of the CCAF.

Experiment 2: This verifies the detection performance of the GPS weak echo signal detection method based on multi-star data fusion for different satellite number conditions, the false alarm probability, GPS direct wave power, and sampling points. Assuming that the target is $10 \mathrm{~km}$ away from the receiver, the speed is $600 \mathrm{~m} / \mathrm{s}$, and the arrival angles $\theta$ of the three echoes are $45^{\circ}, 50^{\circ}, 60^{\circ}$, respectively. It is concluded that the time delay and Doppler shift of the echo relative to the direct wave are $10 \mathrm{us}, 3157 \mathrm{~Hz}, 11 \mathrm{us}, 2669 \mathrm{~Hz}, 16 \mathrm{us}, 2467 \mathrm{~Hz}$, and the echo power is $70 \mathrm{~dB}$ different from the direct wave power. The SNR range of the echo is from $-90 \mathrm{~dB}$ to $-30 \mathrm{~dB}$. For different satellite numbers, 2000 Monte Carlo experiments are carried out on the detection method of the GPS weak echo signal based on data fusion of multiple satellites. Finally, the parameters are substituted into Equations (35) and (42), and the theoretical detection probability is compared with the simulation. The specific simulation parameters are shown in Table 5.

Table 5. Parameter setting of experiment 2.

\begin{tabular}{|c|c|c|c|c|c|c|}
\hline Sampling Frequency $(\mathrm{Hz})$ & Duration (ms) & False Alarm Probability & Number of Satellites & Direct Wave Power (dBm) & Echo Power (dBm) & SNR (dB) \\
\hline $10.23 \mathrm{MHz}$ & $1000 \mathrm{~ms}$ & 0.0001 & $1 / 2 / 3$ & -99.3 & -170 & $-90 \sim-30$ \\
\hline
\end{tabular}

From Figure 15, it can be seen that, under the same conditions, the detection probability of a satellite is $99 \%$ when the echo SNR is $-65 \mathrm{~dB}$, and the detection probability of two satellites is $99 \%$ when the echo SNR is $-71 \mathrm{~dB}$, and the detection probability of three satellites is $99 \%$ when the echo SNR is $-74 \mathrm{~dB}$, and the simulation and the theoretical detection performances are only about $2 \mathrm{~dB}$ in difference. The validity of the method is verified. The above simulation results show that, compared with the traditional single satellite detection, the fusion of multiple satellite detections can effectively 
enhance the detection performance. On the one hand, the algorithm can effectively convert multiple GPS delay-Doppler detection peak coordinates (10 us, 3157 Hz), (11 us, 2669 Hz), (16 us, 2467 Hz) to distance-speed detection peak $(10 \mathrm{~km}, 600 \mathrm{~m} / \mathrm{s})$. Furthermore, a plurality of echo peaks can be superimposed, thereby enhancing the peak value of the echo peak. On the other hand, the method uses the CCAF algorithm to construct the detection amount, which suppresses the noise caused by the cross terms. Therefore, the method enhances the detection performance from two aspects: the cumulative effect of the multi-star detection fusion on the echo peak and the de-noise of the CCAF detection structure. However, it is necessary to consider that there are only 7-8 GPS satellites in the zenith at the same time, and detection performance improves as the number of satellites increases.

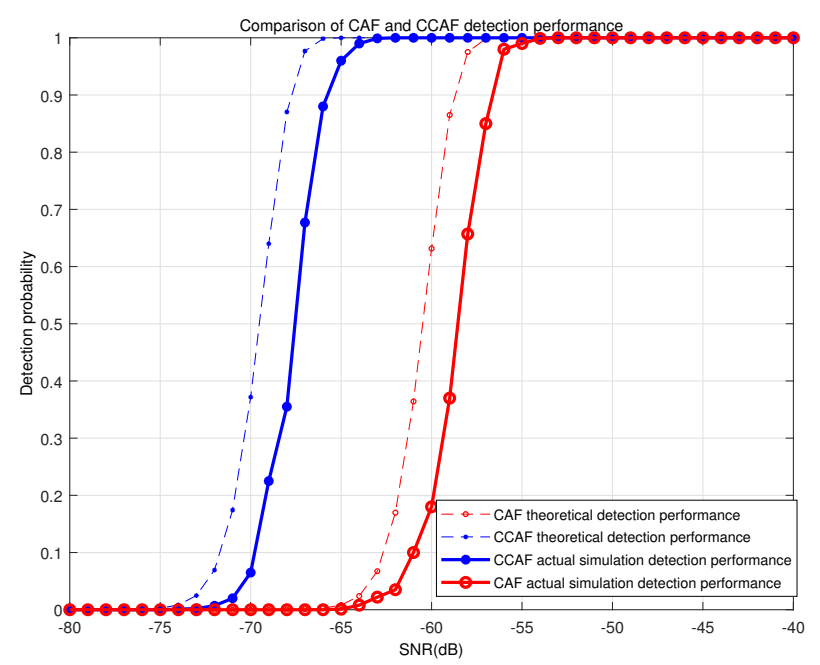

Figure 14. Comparison of detection performance between CCAF and CAF.

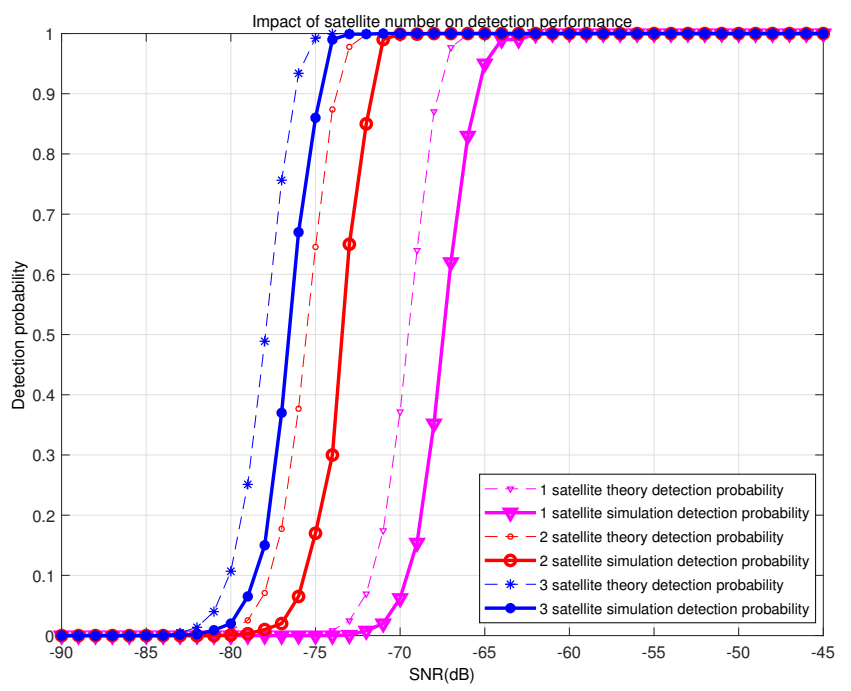

Figure 15. Comparison of detection performance between CCAF and CAF.

Experiment 3: In order to verify the influence of the accumulated time of the signal on the detection performance, we set the parameters of the false alarm probability, the direct wave power of GPS, the number of satellites, etc. The echo parameters are set according to Experiment 2. The SNR of the echo ranges from -90 to $-30 \mathrm{~dB}$. The Monte Carlo experiment was performed on the proposed algorithm under the condition that the number of sampling points changed from $10^{6}$ to $10^{9}$ points. Finally, the parameters are substituted into Equation (35) and Equation (42), and the theoretical 
detection probability is compared with the actual simulation. The specific simulation parameters are shown in Table 6.

Table 6. Parameter setting of experiment 3.

\begin{tabular}{|c|c|c|c|c|c|c|}
\hline Sampling Frequency $(\mathrm{Hz})$ & Duration (ms) & False Alarm Probability & Number of Satellites & Direct Wave Power (dBm) & Echo Power (dBm) & SNR (dB) \\
\hline $10.23 \mathrm{MHz}$ & $0.1 \mathrm{~s} / 1 \mathrm{~s} / 10 \mathrm{~s}$ & 0.0001 & 3 & -110 & -170 & $-90 \sim-30$ \\
\hline
\end{tabular}

From the results in Figure 16, it can be seen that, under the same conditions, in the case of echo SNR of $-60 \mathrm{~dB}$, the detection probability of the cumulative time of $0.1 \mathrm{~s}$ reaches $99 \%$; in the case of echo SNR of $-71 \mathrm{~dB}$, the detection probability of the cumulative time of $1 \mathrm{~s}$ reaches $99 \%$, in the case of echo SNR of $-78 \mathrm{~dB}$, the detection probability of the cumulative time of $10 \mathrm{~s}$ reaches $99 \%$, and the simulated and theoretical detection performances are only $2 \mathrm{~dB}$ in difference, which verifies the effectiveness of the method. From the simulation results, it can be found that, as the accumulation time increases, the detection probability of the algorithm will increase. This is because CCAF is two-dimensional correlation. Therefore, the longer the correlation accumulation time, the larger the energy accumulated by the target echo will be. At the same time, as the accumulation time increases, the noise floor of the detection amount is also suppressed. Therefore, the number of sampling points $N$ is one of the key factors affecting the detection performance of the proposed method. It is possible to increase the detection probability by using a longer accumulation time as much as possible, but the accumulation time cannot be increased indefinitely due to the limited detection area.

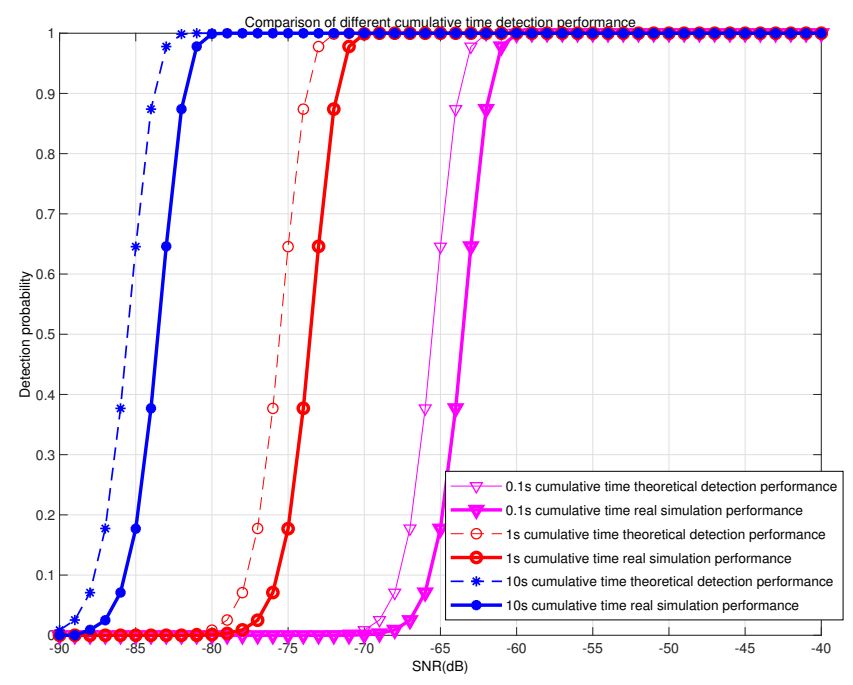

Figure 16. Influence of different accumulation time on detection performance.

Experiment 4: When the detection system detects the target of the specified area, the detection performance is mainly affected by the radar cross section (RCS) area of the target due to the fixed detection distance, and the change of the RCS size causes the change of the direct wave and the echo power ratio (SDR). In order to verify the influence of the attenuation of GPS signal caused by RCS on the detection performance of the proposed algorithm, the experimental fixed false alarm probability, reference signal power, noise power, number of satellites, and sampling points. The echo parameters are set according to the second experiment. Under the condition that the SDR variation range is $105 \mathrm{~dB}$ to $60 \mathrm{~dB}$, the RCS corresponding variation range is $0.01 \mathrm{~m}^{2}$ to $96.3 \mathrm{~m}^{2}$, and 2000 Monte Carlo experiments are performed on the proposed algorithm. Finally, these parameters are substituted into Equations (35) and (42) to compare with the actual simulation. The specific simulation parameters are shown in Table 7. 
Table 7. Parameter setting of experiment 4 .

\begin{tabular}{cccccccc}
\hline Sampling Frequency $(\mathrm{Hz})$ & Duration $(\mathrm{ms})$ & False Alarm Probability & Number of Satellites & Direct Wave Power (dBm) & Noise Power (dBm) & SNR (dB) & Detection Distance (Km) \\
\hline $10.23 \mathrm{MHz}$ & $10 \mathrm{~s}$ & 0.0001 & 3 & -100 & -110 & $-90 \sim-60$ & 10 \\
\hline
\end{tabular}

From the results in Figure 17, it can be seen that the detection probability can reach $99 \%$ when the difference between the direct wave and echo power reaches $80 \mathrm{~dB}$. At the same time, when the detection distance is about $10 \mathrm{~km}$, the weak echo detection algorithm based on the multiple satellites data fusion proposed in this paper can effectively detect the weak GPS signal with an $80 \mathrm{~dB}$ attenuation. Both theoretical analysis and simulation verify that the proposed algorithm can effectively detect close-range targets.

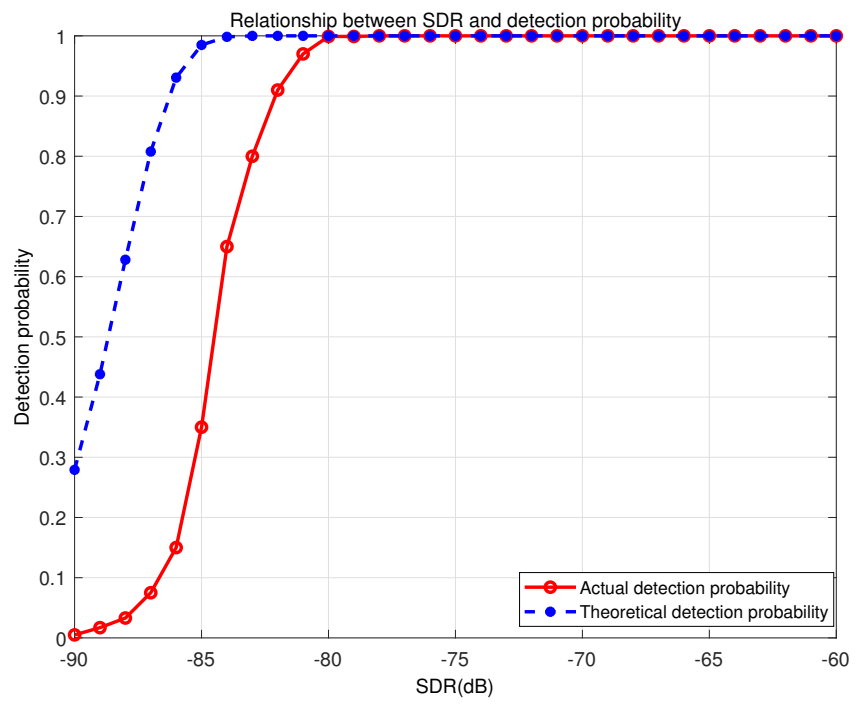

Figure 17. Relationship between SDR and detection probability.

\section{Conclusions}

In this paper, a GPS weak echo signal detection method has been proposed based on data fusion of multiple satellites, in which the SIC algorithm is utilized to separate and reconstruct multiple GPS reference signals on a reference channel. Then, the detection statistic of the weak echo of multiple GPS satellites has been constructed by using the anti-interference property of CCAF. Through the coordinate transformation algorithm, multiple detection statistics have been superimposed to obtain the final detection statistic. Finally, based on the theoretical analysis of the detection statistics, the relationships between the detection performance and a series of key parameters have been studied. Numerical results have shown that the proposed method is proved to be able to effectively detect the weak echo of the target in multiple GPS scenarios and significantly outperforms the existing methods.

Author Contributions: M.L. conceptualized and performed the algorithm and wrote the paper; Z.G. and Y.L. analyzed the experiment data; Y.C. helped modify the language; H.S. provided technical assistance to the research; F.G. is the research supervisor. The manuscript was discussed by all co-authors. All authors have read and agreed to the published version of the manuscript.

Funding: This research was funded by the National Natural Science Foundation of China (61501348 and 61801363), the Shaanxi Provincial Key Research and Development Program (2019GY-043), the Joint Fund of Ministry of Education of the People's Republic of China (6141A02022338), the China Postdoctoral Science Foundation (2017M611912), the 111 Project (B08038), and the China Scholarship Council (201806965031).

Conflicts of Interest: The authors declare no conflict of interest. 


\section{Appendix A}

Appendix A.1. Proof of Lemma 1

Substituting Equations (26) and (27) into Equation (14), we can obtain

$$
\begin{aligned}
S_{i}(\tau, f) & =\sum_{n=0}^{N} x_{s}^{I F}\left(n T_{s}\right) \hat{x}_{i}^{I F}\left(n T_{s}-\tau\right)^{*} e^{j 2 \pi f n T_{s}} \\
& =\sum_{n=0}^{N} \operatorname{Re}\left\{x_{s}^{I F}\left(n T_{s}\right) \hat{x}_{i}^{I F}\left(n T_{s}-\tau\right)^{*} e^{j 2 \pi f n T_{s}}\right\} \\
& +j \sum_{n=0}^{N} \operatorname{Im}\left\{x_{s}^{I F}\left(n T_{s}\right) \hat{x}_{i}^{I F}\left(n T_{s}-\tau\right)^{*} e^{j 2 \pi f n T_{s}}\right\} .
\end{aligned}
$$

Analyzing the probability distribution of the real part in Equation (A1), and the real part in Equation (A1) is given by

$$
\begin{aligned}
\operatorname{Re}\left(S_{i}(\tau, f)\right) & =\sum_{n=0}^{N} \operatorname{Re}\left\{x_{s}^{I F}\left(n T_{s}\right) \hat{x}_{i}^{I F}\left(n T_{s}-\tau\right)^{*} e^{j 2 \pi f n T_{s}}\right\} \\
& =\sum_{n=0}^{N} \operatorname{Re}\left\{\alpha_{i}^{*} \beta_{i} p_{i}\left(n T_{s}-\tau_{i}\right) e^{-j\left(2 \pi f_{d_{i}} n T_{s}\right)} p_{i}^{*}\left(n T_{s}-\tau\right) e^{j 2 \pi f n T_{s}}\right\} \\
& +\sum_{n=0}^{N} \operatorname{Re}\left\{\beta_{i} p_{i}\left(n T_{s}-\tau_{i}\right) e^{-j\left(2 \pi f_{d_{i}} n T_{s}\right)}\left(\sum_{\substack{j=1 \\
\forall i \neq j}}^{M} \alpha_{j} p_{j}\left(n T_{s}-\tau_{j}\right) e^{j 2 \pi f n T_{s}}\right\}\right. \\
& +\sum_{n=0}^{N} \operatorname{Re}\left\{\beta_{i} p_{i}{ }^{*}\left(n T_{s}-\tau\right) e^{j 2 \pi f n T_{s}} \omega\left(n T_{s}\right)\right\} \\
& \approx \sum_{n=0}^{N} \operatorname{Re}\left\{\alpha_{i}{ }^{*} \beta_{i} p_{i}\left(n T_{s}-\tau_{i}\right) e^{-j\left(2 \pi f_{d_{i}} n T_{s}\right)} p_{i}^{*}\left(n T_{s}-\tau\right) e^{j 2 \pi f n T_{s}}\right\} \\
& +\sum_{n=0}^{N} \operatorname{Re}\left\{\beta_{i} p_{i}{ }^{*}\left(n T_{s}-\tau\right) e^{j 2 \pi f n T_{s}} \omega\left(n T_{s}\right)\right\} \\
& =S_{r_{i} s_{i}}(\tau, f)+S_{r_{i} \omega}(\tau, f) .
\end{aligned}
$$

The reason for the sign being approximately equal in Equation (A2) is that the baseband signals of different GPS satellites are not correlated with each other, and the power of the echo signals is much smaller than the noise. The first term $S_{r_{i} s_{i}}(\tau, f)$ in Equation (A2) represents the real part of the mutual ambiguity function between the reference signal and its corresponding echo signal, and belongs to the determined detection amount, which can be expressed as

$$
\begin{aligned}
S_{r_{i} s_{i}}(\tau, f) & =\sum_{n=0}^{N} \operatorname{Re}\left\{\alpha_{i}^{*} \beta_{i} p_{i}\left(n T_{s}-\tau_{i}\right) e^{-j\left(2 \pi f_{d_{i}} n T_{s}\right)} p_{i}^{*}\left(n T_{s}-\tau\right) e^{j 2 \pi f_{d_{i}} n T_{s}}\right\} \\
& =\operatorname{Re}\left\{\alpha^{*} \beta \chi_{p p}\left(\tau-n_{\tau} T_{s}, f-f_{d}\right)\right\},
\end{aligned}
$$

where $n_{\tau}$ stands for the time delay of the echo relative to the direct wave, $f_{d}$ is the frequency offset of the echo relative to the direct wave, and $\chi_{p p}(\tau, f)$ is the self-fuzzy function of the amplitude-normalized baseband signal $p_{i}\left(n T_{s}\right)$, which can be expressed as

$$
\chi_{p p}(\tau, f)=\sum_{n=0}^{N-1} p_{i}\left(n T_{s}\right) p_{i}^{*}\left(n T_{s}-\tau\right) e^{j 2 \pi f n T_{s}},
$$

where $\left|p_{i}\left(n T_{s}\right)\right|=1, E\left[p_{i}\left(n T_{s}\right)\right]=0$. When $\tau=0, f_{d}=0$, the maximum value reached by Equation (A4) is $\max \left\{\left|\chi_{p p}\left(\tau, f_{d}\right)\right|\right\}=N$.

The second term $S_{r_{i} \omega}(\tau, f)$ in Equation (A2) represents the mutual blur function between the monitoring channel noise and the reference signal, which can be regarded as the product of the 
Gaussian noise and the amplitude scaling factor $p_{i}\left(n T_{s}\right)$, and still obeys the Gaussian distribution. Therefore, the mean and variance of the term can represent the probability distribution of the term. The mean of $S_{r_{i} \omega}(\tau, f)$ is given by

$$
E\left\{S_{r_{i} \omega}(\tau, f)\right\}=0
$$

and the variance of $S_{r_{i} \omega}(\tau, f)$ is given by

$$
\begin{aligned}
& \operatorname{Var}\left[S_{r_{i} \omega}(\tau, f)\right] \\
& =E\left[\left(\sum_{n=0}^{N} \operatorname{Re}\left\{\beta_{i} \omega\left(n T_{s}\right) p_{i}^{*}\left(n T_{s}-\tau\right) e^{j 2 \pi f n T_{s}}\right\}\right)^{2}\right]-E\left[\sum_{n=0}^{N} \operatorname{Re}\left\{\beta_{i} \omega\left(n T_{s}\right) p_{i}^{*}\left(n T_{s}-\tau\right) e^{j 2 \pi f n T_{s}}\right\}\right]^{2} \\
& =E\left[\left(\sum_{n=0}^{N} \operatorname{Re}\left\{\beta_{i} \omega\left(n T_{s}\right) p_{i}{ }^{*}\left(n T_{s}-\tau\right) e^{j 2 \pi f n T_{s}}\right\}\right)^{2}\right] \\
& =\sum_{n=0}^{N} \sum_{m=0}^{N} E\left[\operatorname{Re}\left\{\beta_{i} \omega\left(n T_{s}\right) p_{i}^{*}\left(n T_{s}-\tau\right) e^{j 2 \pi f n T_{s}} \cdot \beta_{i} \omega\left(m T_{s}\right)^{*} p_{i}\left(m T_{s}-\tau\right) e^{-j 2 \pi f m T_{s}}\right\}\right] \\
& =\beta_{i}^{2} \sum_{n=0}^{N} \sum_{m=0}^{N} R_{\omega \omega}\left(n T_{s}-m T_{s}\right) \cdot R_{p_{i} p_{i}}\left(n T_{s}-m T_{s}\right) e^{j 2 \pi f(n-m) T_{s}} \\
& =\beta_{i}^{2} \sum_{n=0}^{N} \sigma_{\omega}^{2} \delta(0) R_{p p}(0) \\
& =N \beta_{i}^{2} \sigma_{\omega}^{2}
\end{aligned}
$$

where $R_{\omega \omega}\left(n T_{s}-m T_{s}\right)$ is the autocorrelation of the noise, and $R_{\omega \omega}\left(n T_{s}-m T_{s}\right)=\sigma_{\omega}^{2} \delta\left(n T_{s}-m T_{s}\right)$, $\delta\left(n T_{S}\right)$ is the sequence of unit samples, and $R_{p_{i} p_{i}}\left(n T_{S}-m T_{S}\right)$ is the autocorrelation of the normalized signal. According to Equations (A4)-(A6), under the assumption of $H_{1}$, the distribution of the real part of $S_{i}(\tau, f)$ can be expressed as

$$
\operatorname{Re}\left(S_{i}(\tau, f)\right) \sim N\left(\operatorname{Re}\left\{\alpha_{i}^{*} \beta_{i} \chi_{p p}\left(\tau-n_{\tau}, f-f_{d}\right)\right\}, N \beta_{i}^{2} \sigma_{\omega}^{2}\right) .
$$

The same can be obtained. Under the $H_{1}$ hypothesis, the probability distribution of the imaginary part of $S_{i}\left(\tau, f_{d}\right)$ can be expressed as

$$
\operatorname{Im}\left(S_{i}(\tau, f)\right) \sim N\left(\operatorname{Im}\left\{\alpha_{i}^{*} \beta_{i} \chi_{p p}\left(\tau-n_{\tau}, f-f_{d}\right)\right\}, N \beta_{i}^{2} \sigma_{\omega}^{2}\right) .
$$

Therefore, the distribution of the detection statistic under the $H_{1}$ hypothesis is

$$
\left(S_{i}(\tau, f) \mid H_{1}\right) \sim C N\left(\left\{\alpha_{i}^{*} \beta_{i} \chi_{p p}\left(\tau-n_{\tau}, f-f_{d}\right)\right\}, \quad 2 N \beta_{i}^{2} \sigma_{\omega}^{2}\right),
$$

where $C N($.$) represents the complex Gaussian process.$

Appendix A.2. Proof of Lemma 2

First, the reference signal $\hat{x}_{i}^{I F}\left(n T_{s}\right)$ is cyclically autocorrelated by

$$
\begin{aligned}
R_{r_{i} r_{i}}^{\alpha}(\tau) & =\frac{1}{N} \sum_{n=0}^{N-1} \hat{x}_{i}^{I F}\left(n T_{S}+\tau / 2\right) \hat{x}_{i}^{I F}\left(n T_{S}-\tau / 2\right)^{*} e^{-j 2 \pi \alpha n T_{s}} \\
= & \beta_{i}^{2} R_{p_{i} p_{i}}^{\alpha}(\tau)
\end{aligned}
$$

where $R_{r_{i} r_{i}}^{\alpha}(\tau)$ is the cyclic autocorrelation of two reference signals, $\alpha$ is the cyclic frequency, and $R_{p_{i}}^{\alpha} p_{i}(\tau)$ stands for the cyclic autocorrelation normalized by the amplitudes of the two reference signals, which can be expressed as

$$
R_{p_{i} p_{i}}^{\alpha}(\tau)=\frac{1}{N} \sum_{n=0}^{N-1}\left[C_{i}\left(n T_{s}+\tau / 2\right) \cdot D_{i}\left(n T_{s}+\tau / 2\right)\right] \cdot\left[C_{i}\left(n T_{s}-\tau / 2\right) \cdot D_{i}\left(n T_{s}-\tau / 2\right)\right]^{*} e^{-j 2 \pi \alpha n T_{s}} .
$$


Then, the reference signal $\hat{x}_{i}^{I F}\left(n T_{s}\right)$ and the echo signal $x_{s}^{I F}\left(n T_{s}\right)$ are subjected to cyclic cross-correlation processing under the assumption of $H_{1}$. In addition, using the uncorrelated properties of the baseband GPS reference signal pseudo-random code to obtain

$$
\begin{aligned}
R_{r_{i} s}^{\alpha-f}(\tau) & =\frac{1}{N} \sum_{n=0}^{N-1} \hat{x}_{i}^{I F}\left(n T_{s}+\tau / 2\right) x_{s}^{I F}\left(n T_{s}-\tau / 2\right)^{*} e^{-j 2 \pi(\alpha-f) n T_{s}} \\
& =\frac{1}{N} \sum_{n=0}^{N-1}\left[\beta_{i} p_{i}\left(n T_{s}\right)\right] \cdot\left[\sum_{\substack{j=1 \\
i \in j}}^{M} \alpha_{j} p_{j}\left(n T_{s}-\tau_{j}\right) e^{j 2 \pi f_{d_{j}} n T_{s}}+\omega\left(n T_{s}\right)\right]^{*} e^{-j 2 \pi(\alpha-f) n T_{s}} \\
& =\frac{1}{N} \sum_{n=0}^{N-1}\left[\beta_{i} p_{i}\left(n T_{s}\right)\right] \cdot\left[\alpha_{i} p_{i}\left(n T_{s}-\tau_{i}\right) e^{j 2 \pi f_{d_{i}} n T_{s}}+\omega\left(n T_{s}\right)\right]^{*} e^{-j 2 \pi(\alpha-f) n T_{s}} \\
& =\beta_{i} \alpha_{i} e^{-j \pi f_{d_{i}} \tau} e^{-j \pi\left(\alpha-f+f_{d_{i}}\right) \tau_{i}} R_{p_{i} p_{i}}^{\alpha-f+f_{d_{i}}}\left(\tau-\tau_{i}\right)+N^{\alpha}(\tau),
\end{aligned}
$$

where $\tau_{i}$ is the time delay of the echo relative to the direct wave, $f_{d_{i}}$ is the Doppler shift of the echo relative to the direct wave, and $N^{\alpha}(\tau)$ is the cyclic cross-correlation of the reference signal $\hat{x}_{i}^{I F}\left(n T_{s}\right)$ and the monitoring channel noise $\omega\left(n T_{S}\right)$, which can be expressed as follows:

$$
N^{\alpha}(\tau)=\frac{1}{N} \sum_{n=0}^{N-1} \hat{x}_{i}^{I F}\left(n T_{s}+\tau / 2\right) \omega\left(n T_{s}-\tau / 2\right)^{*} e^{-j 2 \pi(\alpha-f) n T_{s}}
$$

Equation (A13) obeys a Gaussian distribution with a variance of $\frac{1}{N} \beta_{i}^{2} \sigma_{\omega}^{2}$ and a mean of 0 , which is denoted here by $N^{\alpha}(\tau)$. It can be seen that $R_{r_{i} s}^{\alpha-f}(\tau)$ is obtained by delay and frequency offset of $R_{r_{i} r_{i}}^{\alpha}(\tau)$, and the delay and frequency offset of $R_{r_{i} s}^{\alpha-f}(\tau)$ with respect to $R_{r_{i} r_{i}}^{\alpha}(\tau)$ is just the delay and frequency offset of the echo. Next, the column vectors corresponding to the cyclic frequencies of the maximum peaks of $R_{r_{i} r_{i}}^{\alpha}(\tau)$ and $R_{r_{i} s}^{\alpha-f}(\tau)$ are extracted, denoted respectively as $R_{r_{i} s}^{\alpha^{\prime}-f^{\prime}}(\tau)$ and $R_{r_{i} r_{i}}^{\alpha^{\prime}}(\tau)$, and subjected to mutual blur function processing to obtain

$$
\begin{aligned}
\Psi_{i}(u, f) & =\sum_{\tau=0}^{N} R_{r_{i} s}^{\alpha^{\prime}-f^{\prime}}(\tau) R_{r_{i} r_{i}}^{\alpha^{\prime}}(\tau-u)^{*} e^{j 2 \pi f \tau} \\
& =\beta_{i}^{3} \alpha_{i} e^{-j \pi \alpha^{\prime} \tau_{i}} \sum_{\tau=0}^{N} R_{p_{i} p_{i}}^{\alpha^{\prime}-f+f_{d_{i}}}\left(\tau-\tau_{i}\right) R_{p_{i} p_{i}}^{\alpha^{\prime}}(\tau-u)^{*} e^{j 2 \pi\left(f-f_{d_{i}}\right) \tau} \\
& +\beta_{i}^{2} \sum_{\tau=0}^{N} N^{\alpha^{\prime}}(\tau) R_{p_{i} p_{i}}^{\alpha^{\prime}}(\tau-u)^{*} e^{j 2 \pi f \tau} \\
& =\Psi_{R_{i} R_{i}}(u, f)+\Psi_{N R_{i}}(u, f),
\end{aligned}
$$

where $\Psi_{R, R_{i}}(u, f) \leq \beta_{i}^{3} \alpha_{i} \int\left|R_{p_{i} p_{i}}^{\alpha^{\prime}}(\tau)\right|^{2} d \tau$. When $u=\tau_{i}$ and $f=f_{d_{i}}$,

$$
\Psi_{R_{i} R_{i}}(u, f)=\beta_{i}^{3} \alpha_{i} \int\left|R_{p_{i} p_{i}}^{\alpha^{\prime}}(\tau)\right|^{2} d \tau,
$$

where $\Psi_{N R_{i}}(u, f)$ is the noise term. According to the principle of constant false alarm detection, the size of the detection threshold is related to the false alarm probability. Therefore, the probability distribution of the detection quantity $\Psi_{i}(u, f)$ needs to be analyzed under the assumption of $H_{1}$. The first term of Equation (A14) belongs to the determined detection amount and is expressed as

$$
\Psi_{R_{i} R_{i}}(u, f)=\beta_{i}^{3} \alpha_{i} e^{-j \pi \alpha^{\prime} \tau_{i}} \sum_{\tau=0}^{N} R_{p_{i} p_{i}}^{\alpha^{\prime}-f+f_{d_{i}}}\left(\tau-\tau_{i}\right) R_{p_{i} p_{i}}^{\alpha^{\prime}}(\tau-u)^{*} e^{j 2 \pi\left(f-f_{d_{i}}\right) \tau} .
$$


For the second term of Equation (A14), this term represents the mutual fuzzy function of the cyclic autocorrelation of the reference channel and the noise of the echo channel. If the cycle frequency is $\alpha^{\prime}$, the term can be expressed as

$$
\Psi_{N R_{i}}(u, f)=\beta_{i}^{2} \sum_{\tau=0}^{N} N^{\alpha^{\prime}}(\tau) R_{p_{i} p_{i}}^{\alpha^{\prime}}(\tau-u)^{*} e^{j 2 \pi f \tau}
$$

where $N^{\alpha^{\prime}}(\tau)$ obeys a Gaussian distribution with a mean of 0 and a variance of $\frac{1}{N} \beta_{i}^{2} \sigma_{\omega}^{2}$, and $R_{p_{i}}^{\alpha_{i}^{\prime}}$ is a cyclic autocorrelation of the signal. Since the term is obtained by linear integral operation on noise, the term is still subject to Gaussian distribution, so the mean and variance can be used to characterize the probability distribution. The mean and variance are given by

$$
\begin{aligned}
E\left\{\Psi_{N R_{i}}(u, f)\right\}=0, & \\
\operatorname{Var}\left\{\psi_{N R_{i}}(u, f)\right\} & =E\left\{\left|\psi_{N R_{i}}(u, f)\right|^{2}\right\}-E\left\{\psi_{N R_{i}}(u, f)\right\}^{2} \\
= & E\left\{\left[\beta_{i}^{2} \sum_{\tau_{1}=0}^{N-1} N^{\alpha^{\prime}}\left(\tau_{1}-u\right) R_{p_{i} p_{i}}^{\alpha^{\prime}}\left(\tau_{1}\right) e^{j \pi f \tau_{1}}\right] *\left[\beta_{i}^{2} \sum_{\tau_{2}=0}^{N-1} N^{\alpha^{\prime}}\left(\tau_{2}-u\right) R_{p_{i} p_{i}}^{\alpha^{\prime}}\left(\tau_{2}\right) e^{j \pi f \tau_{2}}\right]^{*}\right\} \\
= & \beta_{i}^{4} \sum_{\tau_{1}=0}^{N-1} \sum_{\tau_{2}=0}^{N-1} E\left\{N^{\alpha^{\prime}}\left(\tau_{1}-u\right) R_{p_{i} p_{i}}^{\alpha^{\prime}}\left(\tau_{1}\right) e^{j \pi f \tau_{1}} *\left(\sum_{\tau_{2}=0}^{N-1} N^{\alpha^{\prime}}\left(\tau_{2}-u\right) R_{p_{i} p_{i}}^{\alpha^{\prime}}\left(\tau_{2}\right) e^{j \pi f \tau_{2}}\right)^{*}\right\} \\
= & \beta_{i}^{4} \sum_{\tau_{1}=0}^{N-1} \sum_{\tau_{2}=0}^{N-1} R_{N N}\left(\tau_{1}-\tau_{2}\right) E\left\{R_{p_{i} p_{i}}^{\alpha^{\prime}}\left(\tau_{1}\right) R_{p_{i} p_{i}}^{\alpha^{\prime *}}\left(\tau_{2}\right) e^{j \pi f\left(\tau_{1}-\tau_{2}\right)}\right\} \\
= & \beta_{i}^{4} \sum_{\tau_{1}=0}^{N-1} \sum_{\tau_{2}=0}^{N-1} \frac{1}{N} \beta_{i}^{2} \sigma_{\omega}^{2} \delta\left(\tau_{1}-\tau_{2}\right) E\left\{R_{p_{i} p_{i}}^{\alpha^{\prime}}\left(\tau_{1}\right) R_{p_{i} p_{i}}^{\alpha^{\prime *}}\left(\tau_{2}\right) e^{j \pi f\left(\tau_{1}-\tau_{2}\right)}\right\} \\
= & \frac{\sigma_{\omega}^{2} \beta_{i}^{6}}{N} .
\end{aligned}
$$

Therefore, the distribution of $\Psi_{N R_{i}}(u, f)$ can be expressed as $\Psi_{N R_{i}}(u, f) \sim C N\left(0, \frac{\sigma_{\omega}^{2} \beta_{i}^{6}}{N}\right)$. It can be seen that the variance of the noise of the item is inversely proportional to the number of sampling points. Obviously, as the number of sampling points increases, the variance of the noise decreases, showing good noise suppression performance. Thus, the distribution of $\Psi_{i}(u, f)$ under the assumption of $H_{1}$ is given by

$$
\Psi_{i}\left((u, f) \mid H_{1}\right) \sim C N\left(\Psi_{R_{i} R_{i}}(u, f), \frac{\sigma_{\omega}^{2} \beta_{i}^{6}}{N}\right) .
$$

\section{References}

1. Foged, L.J.; Saporetti, M.; Scialacqua, L. Measurement and Simulation Comparison Using Measured Source Antenna Representation of GNSS Antenna on Sentinel Satellite. In Proceedings of the 2017 IEEE International Symposium on Antennas and Propagation USNC/URSI National Radio Science Meeting, San Diego, CA, USA, 9-15 July 2017; pp. 1933-1934.

2. Powell, S.J.; Akos, D.M. GNSS Reflectrometry Using the L5 and E5a Signals for Remote Sensing Applications. In Proceedings of the 2013 US National Committee of URSI National Radio Science Meeting, Boulder, CO, USA, 9-12 January 2013; p. 1.

3. Giangregorio, G.; Bisceglie, M.; Addabbo, P.; Beltramonte, T.; D'Addio, S.; Galdi, C. Stochastic modeling and simulation of Delay-Doppler Maps in GNSS-R over the ocean. IEEE Trans. Geosci. Remote Sens. 2016, 54, 2056-2069. [CrossRef]

4. Fernez-Prades, C.; Arribas, J.; Majoral, M.; Ramos, A.; Vilá-Valls, J.; Giordano, P. A Software-Defined Spaceborne GNSS Receiver. In Proceedings of the 2018 9th ESA Workshop on Satellite NavigationTechnologies and European Workshop on GNSS Signals and Signal Processing, Noordwijk, The Netherlands, 5-7 December 2018; pp. 1-9. 
5. Imam, R.; Pini, M.; Marucco, G.; Dominici, F.; Dovis, F. Data from GNSS-Based Passive Radar to Support Flood Monitoring Operations. In Proceedings of the 2019 International Conference on Localization and GNSS, Nuremberg, Germany, 4-6 June 2019; pp. 1-7.

6. Khosravi, F.; Moghadas, H.; Mousavi, P. A GNSS Antenna with a Polarization Selective Surface for the Mitigation of Low-Angle Multipath Interference. IEEE Trans. Antennas Propag. 2015, 63, 5287-5295. [CrossRef]

7. Liu, F.; Antoniou, M.; Zeng, Z.; Cherniakov, M. Coherent change detection using passive GNSS-based BSAR: Experimental proof of concept. IEEE Trans. Geosci. Remote Sens. 2013, 51, 4544-4555. [CrossRef]

8. Qiao, J.; Chen, W.; Ji, S.; Weng, D. Accurate and Rapid Broadcast Ephemerides for Beidou-Maneuvered Satellites. Remote Sens. 2019, 11, 787. [CrossRef]

9. Yan, S.; Chen, N.; Gong, J.; Zhang, X. Sea State Sensing with Single Frequency GPS Receiver. In Proceedings of the 2011 IEEE International Geoscience and Remote Sensing Symposium, Vancouver, BC, Canada, 24-29 July 2011; pp. 2073-2076.

10. Clarizia, M.P.; Chotiros, N.P.; Vaccaro, M.G. A GPS-Reflectometry Simulator for Target Detection Over Oceans. In Proceedings of the IGARSS 2018-2018 IEEE International Geoscience and Remote Sensing Symposium, Valencia, Spain, 22-27 July 2018; pp. 450-451.

11. Garvanov, I.; Kabakchiev, C.; Behar, V.; Garvanova, M. Target Detection Using a GPS Forward-Scattering Radar. In Proceedings of the 2015 International Conference on Engineering and Telecommunication, Mosocw, Russia, 18-19 November 2015; pp. 29-33.

12. Clarizia, M.; Braca, P.; Ruf, C.S.; Willett, P. Target Detection Using GPS Signals of Opportunity. In Proceedings of the 2015 18th International Conference on Information Fusion, Washington, DC, USA, 6-9 July 2015; pp. 1429-1436.

13. Gronowski, K.; Samczyński, P.; Stasiak, K.; Kulpa, K. First, Results of Air Target Detection Using Single Channel Passive Radar Utilizing GPS Illumination. In Proceedings of the 2019 IEEE Radar Conference (RadarConf), Boston, MA, USA, 22-26 April 2019; pp. 1-6.

14. Mojarrabi, B.; Homer, J.; Kubik, K. Power Budget Study for Passive Target Detection and Imaging Using Secondary Applications of GPS Signals in Bistatic Radar Systems. In Proceedings of the IEEE International Geoscience and Remote Sensing Symposium, Toronto, ON, Canada, 24-28 June 2002; pp. 449-451.

15. AlJewari, Y.H.; Ahmad, R.B.; AlRawi, A.A. Impact of multipath interference and change of velocity on the reliability and precision of GPS. In Proceedings of the 2014 2nd International Conference on Electronic Design (ICED), Penang, Malaysia, 19-21 August 2014; pp. 427-430.

16. Wu, X.; Gong, P.; Zhou, J.; Liu, Z. The applied research on anti-multipath interference GPS signal based on narrow-related. In Proceedings of the 2014 IEEE 5th International Conference on Software Engineering and Service Science, Beijing, China, 27-29 June 2014; pp. 771-774.

17. Suberviola, I.; Mayordomo, I.; Mendizabal, J. Experimental Results of Air Target Detection with a GPS Forward-Scattering Radar. IEEE Geosci. Remote Sens. Lett. 2011, 9, 47-51. [CrossRef]

18. Lashley, M.; Bevly, D.; Hung, J. Performance Analysis of Vector Tracking Algorithms for Weak GPS Signals in High Dynamics. IEEE J. Sel. Top. Signal Process. 2009, 3, 661-673. [CrossRef]

19. Wang, L.; Wang, J.; Xiao, L. Passive Location and Precision Analysis Based on Multiple CDMA Base Stations. In Proceedings of the 2009 IET International Radar Conference, Guilin, China, 20-22 April 2009; pp. 1-4.

20. Michael, E.; Alexander, S.; Fabienne, M. Design and Performance Evaluation of a MatureFM/DAB/DVB-T Multi-illuminator Passive Radar System. IET Radar Sonar Navig. 2014, 8, 114-122.

21. Stephen, D.; Songsri, S. Passive Radar Detection Using Multiple Ransmitters. In Proceedings of the 2013 Asilomar Conference on Signals Systems and Computers, Pacific Grove, CA, USA, 3-6 November 2013; pp. 945-948.

22. Zhang, Y.; Xi, S. Application of New LMS Adaptive Filtering Algorithm with Variable Step Size in Adaptive Echo Cancellation. In Proceedings of the 2017 IEEE 17th International Conference on Communication Technology, Chengdu, China, 27-30 October 2017; pp. 1715-1719.

23. Niranjan, D.; Ashwini, B. Noise Cancellation in Musical Signals Using Adaptive Filtering Algorithms. In Proceedings of the 2017 International Conference on Innovative Mechanisms for Industry Applications, Bangalore, India, 21-23 February 2017; pp. 82-86. 
24. Mugdha, A.C.; Rawnaque, F.S.; Ahmed, M.U. A Study of Recursive Least Squares (RLS) Adaptive Filter Algorithm in Noise Removal from ECG Signals. In Proceedings of the 2015 International Conference on Informatics, Electronics Vision, Fukuoka, Japan, 15-18 June 2015; pp. 1-6.

25. Liu, J.; Liu, L. GPS C/A Code Signal Simulation Based on MATLAB. In Proceedings of the 2011 First, International Conference on Instrumentation, Measurement, Computer, Communication and Control, Beijing, China, 21-23 October 2011; pp. 4-6.

26. Beldjilali, B.; Benadda, B. Real Time Software Based L1 C/A GPS receiver. In Proceedings of the 2017 Seminar on Detection Systems Architectures and Technologies, Algiers, Algeria, 20-22 February 2017; pp. 1-8.

27. Ke, Z.; Ying, S. Steady-state Kalman Fusion Filter Based on Improved Multi-innovation Least Squares Algorithm. In Proceedings of the 2018 37th Chinese Control Conference, Wuhan, China, 25-27 July 2018; pp. 4434-4437.

28. Yang, Y.H.; Shi, Y. Iterative Least Squares Method Based Fusion Kalman Filter for Unknown Model Parameters System. In Proceedings of the 2018 37th Chinese Control Conference, Wuhan, China, 25-27 July 2018; pp. 1628-1631.

29. Ballal, T.; Suliman, M.A.; Al-Naffouri, T.Y. Bounded Perturbation Regularization for Linear Least Squares Estimation. IEEE Access 2017, 5, 27551-27562. [CrossRef]

30. He, H.; Stoica, P.; Li, J. On Synthesizing Cross Ambiguity Functions. In Proceedings of the 2011 IEEE International Conference on Acoustics, Speech and Signal Processing, Prague, Czech Republic, 22-27 May 2011; pp. 3536-3539.

31. Huang, L.; Zhang, Y.; Li, Q.; Pan, C.; Song, J. Fast Calculation for Cross Ambiguity Function in Passive Detection Systems. In Proceedings of the 2018 International Conference on Sensing, Diagnostics, Prognostics, and Control, Xi'an, China, 15-17 August 2018; pp. 690-694.

32. Li, J.; He, Y.; Song, J. The Algorithms and Performance Analysis of Cross Ambiguity Function. In Proceedings of the 2009 IET International Radar Conference, Guilin, China, 20-22 April 2009; pp. 1-4.

33. Zeng, H.; Chen, J.; Wang, P.; Yang, W.; Liu, W. 2D Coherent Integration Processing and Detecting of Aircrafts Using GNSS-Based Passive Radar. Remote Sens. 2018, 10, 1164. [CrossRef]

34. Liu, M.; Yi, F.; Liu, P.; Li, B. Cramer-Rao Lower Bounds of TDOA and FDOA Estimation Based on Satellite Signals. In Proceedings of the 2018 14th IEEE International Conference on Signal Processing (ICSP), Beijing, China, 12-16 August 2018; pp. 1-4.

35. Liu, M.; Zhang, J.; Tang, J.; Jiang, F.; Liu, P.; Gong, F.; Zhao, N. 2D DOA Robust Estimation of Echo Signals Based on Multiple Satellites Passive Radar in the Presence of Alpha Stable Distribution Noise. IEEE Access 2019, 7, 2169-3536.

(C) 2020 by the authors. Licensee MDPI, Basel, Switzerland. This article is an open access article distributed under the terms and conditions of the Creative Commons Attribution (CC BY) license (http:/ / creativecommons.org/licenses/by/4.0/). 